FEDERAL

RESERVE

BANK of

ST. LOUIS
RESEARCH DIVISION

Working Paper Series

\title{
Sentiments and Aggregate Demand Fluctuations
}

\author{
Jess Benhabib, \\ Pengfei Wang \\ and \\ Yi Wen
}

\author{
Working Paper 2012-039B \\ https://doi.org/10.20955/wp.2012.039
}

September 2014

\author{
FEDERAL RESERVE BANK OF ST. LOUIS \\ Research Division \\ P.O. Box 442 \\ St. Louis, MO 63166
}

The views expressed are those of the individual authors and do not necessarily reflect official positions of the Federal Reserve Bank of St. Louis, the Federal Reserve System, or the Board of Governors.

Federal Reserve Bank of St. Louis Working Papers are preliminary materials circulated to stimulate discussion and critical comment. References in publications to Federal Reserve Bank of St. Louis Working Papers (other than an acknowledgment that the writer has had access to unpublished material) should be cleared with the author or authors. 


\title{
Sentiments and Aggregate Demand Fluctuations*
}

\author{
Jess Benhabib ${ }^{\dagger} \quad$ Pengfei Wang ${ }^{\ddagger} \quad$ Yi Wen ${ }^{\S}$
}

(This version: September 3, 2014)

\begin{abstract}
We formalize the Keynesian insight that aggregate demand driven by sentiments can generate output fluctuations under rational expectations. When production decisions must be made under imperfect information about demand, optimal decisions based on sentiments can generate stochastic self-fulfilling rational expectations equilibria in standard economies without persistent informational frictions, externalities, non-convexities or strategic complementarities in production. The models we consider are deliberately simple, but could serve as benchmarks for more complicated equilibrium models with additional features.
\end{abstract}

Keywords: Keynesian Self-fulfilling Equilibria, Sentiments, Sunspots

\section{Introduction}

We construct a class of models to capture the Keynesian insight that employment and production decisions are based on expected consumer demand, and that realized aggregate demand follows firms' production and employment decisions. Because of imperfect information in forecasting demand, consumer sentiments can matter in determining equilibrium aggregate supply. We cast the Keynesian insight in a simple dynamic stochastic general equilibrium model and characterize the rational expectations equilibria of this model. We find that despite the lack of any externalities or

\footnotetext{
${ }^{*}$ We are indebted to George-Maria Angeletos, Larry Christiano, George Evans, Jean-Michel Grandmont, Boyan Jovanovic, Guy Laroque, Gaetano Gaballo, John Leahy, Jennifer Lao, Stephen Morris, Heraklis Polemarchakis, Edouard Schaal, Martin Schneider, Karl Shell, Michal Lukasz Szkup, Laura Veldkamp and Michael Woodford for very enlightening comments. We would like to thank the participants at the conference organized by the International Network on Expectational Cordination at the College de France on June 27-29, 2012 in Paris, and at the NorthwesternTsinghua Conference on "Financial Frictions, Sentiments and Aggregate Fluctuations" on August 21-24 2012 in Beijing for their valuable insights. In particular, we are grateful for discussions with Gaetano Gaballo which were very helpful. Wang acknowledges the financial support from the Research Grants Council of Hong Kong under project number 693513.

${ }^{\dagger}$ Department of Economics, New York University, 19 West 4th Street, New York, NY 10012, USA. Office: (212) 998-8971. Fax: (212) 995-4186. Email: jess.benhabib@nyu.edu.

${ }^{\ddagger}$ Department of Economics, The Hong Kong University of Science and Technology, Clear Water Bay, Hong Kong. Office: (+852) 2358 7612. Email: pfwang@ust.hk

${ }^{\S}$ Federal Reserve Bank of St. Louis, P.O. Box 442, St. Louis, MO 63166; and School of Economics and Management, Tsinghua University, Beijing, China. Office: (314) 444-8559. Fax: (314) 444-8731. Email: yi.wen@stls.frb.org.
} 
non-convexities in technology or preferences there can be multiple rational expectations equilibria. Fluctuations can be driven by waves of optimism or pessimism, or as in Keynes' terminology, by "animal spirits" that are distinct from fundamentals. Sentiment-driven equilibria exist because firms must make production decisions prior to the realization of demand, and households must make labor supply decisions and consumption plans before the realization of production. When firm decisions are based on expected demand and household decisions are based on expected income, equilibrium output can be affected by consumer sentiments. A distinctive feature of our results is that in the sentiment driven equilibrium the underlying distribution of household sentiments is pinned down endogenously by deep structural parameters, and corresponds to the self-fulfilling distribution of actual aggregate output.

Our models are in the spirit of the Lucas (1972) island model, as well as the models with sentiment-driven fluctuations of Angeletos and La'O (2009, 2012). In the absence of sentiments, the models that we study have a unique equilibrium, but sentiments and beliefs about aggregate income can affect consumption, which in turn can affect and amplifies employment and production decisions, leading to multiple self-fulfilling stochastic rational expectations equilibria. ${ }^{1,2}$

More specifically, we study models where firms produce differentiated goods (analogous to the islands in the Lucas model), and make production and employment decisions based on imperfect signals about the demand for their goods. Trades take place in centralized markets, and at the end of each period all trading and price history is public knowledge. Consumer demand reflects fundamental idiosyncratic preference shocks to differentiated goods, as well as consumer sentiments about expected aggregate income. The firms cannot precisely distinguish firm-level demand and aggregate demand in their noisy signals and therefore face a signal extraction problem - because their optimal response to idiosyncratic demand shocks is different from their optimal response to aggregate sentiment changes. We show that the signal extraction problem of firms can give rise to sentiment-driven equilibria, and in certain cases a continuum of them, in addition to equilibria solely driven by fundamentals. Such sentiment-driven equilibria are stochastic in nature and can be serially correlated over time, and are not based on randomizations over the fundamental equilibria. ${ }^{3}$

The multiplicity of rational expectations equilibria that we obtain is related to the correlated

\footnotetext{
${ }^{1}$ For the possibility of multiple equilibria in the context of asymmetic information see Amador and Weill (2010), Angeletos and Werning (2006), Angeletos, Hellwig, and Pavan (2006), Angeletos, Lorenzoni and Pavan (2010), Gaballo (2012), Hellwig, Mukherji, and Tsyvinski (2006), and Hellwig and Veldkamp (2009). In particular Manzano and Vives (2010) survey the literature and study the emegerce of multiplicity when correlated private information induces strategic compementarity in the actions of agents trading in financial markets. In a number of the papers cited, prices convey noisy information about asset returns. By contrast in our model production and employment decisions are made based on expectations, but prior to the realization of demand and real prices.

${ }^{2}$ See also Morris and Shin (2002) and Angeletos and Pavan (2007) where agents can excessively coordinate on and overreact to public information, thereby magnifying the fluctuations caused by pure noise. By contrast, in some global games, multiple coordination equilibria may be eliminated under dispersed private signals on fundamentals, as in Morris and Shin (1998).

${ }^{3}$ For the classical work on extrinsic uncertainty and sunspot equilibria with a unique fundamental equilibrium under incomplete markets, see Cass and Shell (1983). See also Spear (1989) for an OLG model with two islands where prices in one island act as sunspots for the other and vice versa.
} 
equilibria of Aumann $(1974,1987)$ and of Maskin and Tirole (1987). ${ }^{4}$ They emerge naturally from the endogenous signals that induce imperfectly correlated employment and output decisions by firms. ${ }^{5,6}$ In equilibrium there exists a distribution of sentiments such that for every realization of the sentiment shocks, the firms' expected aggregate demand is equal to the realized aggregate demand, the consumer's expected aggregate income is equal to the realized aggregate output, and the expected prices and real wages are equal to the realized prices and real wages.

The rest of the paper is organized as follows. Section 2 presents a simple benchmark model, defines rational expectations equilibrium, and then characterizes the fundamental equilibrium and the sentiment-driven equilibrium. In section 2.6, we provide a more abstract and streamlined model further illustrate the mechanisms behind our results. In section 2.7 we show that the fundamental equilibrium is not stable under constant gain learning, while sentiment-driven equilibrium is stable if the gain parameter is not too large. In section 3 we provide explicit microfoundations for the signal and information structures that we consider throughout the paper. Section 4 extends our analysis to other settings and Section 5 concludes.

\section{The Benchmark Model}

The key feature of our model is that production and employment decisions by firms, and consumption and labor supply decisions by households, are made prior to goods being produced and exchanged and before market clearing prices are realized. To provide an early road-map, we start by describing the sequence of actions by consumers and firms, the information structure, and the rational expectations equilibria of our benchmark model.

1. At the beginning of each period, households form expectations on aggregate output/income based on their sentiments. They also form demand functions for each differentiated good based on their sentiments and the idiosyncratic preference shocks on each good, contingent on the prices to be realized when the goods markets open.

2. Like households, firms also believe that aggregate output/demand could be driven by sentiments. Unlike households, firms do not directly observe households' sentiments or idiosyncratic preference shocks. Firms instead receive a noisy signal about their demand, which is

\footnotetext{
${ }^{4}$ Correlated equilibria in market economies are also discussed by Aumann, Peck and Shell (1988). See also Peck and Shell (1991), Forges and Peck (1995), Forges (2006), and more recently, Bergemann and Morris (2011) and Bergemann, Morris and Heinmann (2013).

${ }^{5}$ As noted by Maskin and Tirole (1987), "Our observation that signals "matter" only if they are imperfectly correlated corresponds to the game theoretic principle that perfectly correlated equilibrium payoff vectors lie in the convex hull of the ordinary Nash equilibrium payoffs, but imperfectly correlated equilibrium payoffs need not." In Maskin and Tirole (1987) however the uninformed agents do not have a signal extraction problem as we do, so in their model in addition to the certainty Nash equilibrium, they have correlated equilibria only if there are Giffen goods.

${ }^{6}$ Correlated equilibria are typically defined for finite games with a finite number of agents and discrete strategy sets, but for an extention to continuous games see Hart and Schmeidler (1989) and more recently Stein, Parillo, and Ozdaglar (2008). We thank Martin Schneider for alerting us to this point.
} 
a mixture of firm-specific demand (idiosyncratic preference shocks) and aggregate demand (sentiments).

3. Given a nominal wage, households make labor supply decisions based on their sentiments, and firms make employment and production decisions based on their signals, taking the expected real wage and prices as given. ${ }^{7}$

4. Goods markets open and goods are exchanged at market clearing prices, the real wage and actual consumption are realized.

We show that there exist two equilibria, depending on the distribution of sentiments (beliefs) about aggregate output/income: (i) A fundamental equilibrium with a degenerate distribution of sentiments, where aggregate output and prices are all constant. In this case sentiments play no role in determining the level of aggregate output. (ii) A stochastic equilibrium with positive variance of sentiments. In this case sentiments matter and the variance of sentiments is endogenously determined and consistent with the self-fulfilling variance of aggregate output.

Each of these two equilibria constitutes a rational expectations equilibrium in the sense that for any realization of the sentiment shock, (i) the labor demanded by firms and supplied by households, based on expected real wages, will be equal, (ii) the goods demanded by households and supplied by firms, based on expected prices, will be equal, (iii) the expected aggregate output based on consumer sentiments will be equal to the realized aggregate output produced by firms conditioned on their signals for demand, and the expected prices and real wages will be equal to the realized prices and real wages.

The fundamental equilibrium is unique in our model, so the sentiment-driven stochastic equilibrium is not based on randomization over multiple fundamental equilibria with an arbitrary variance. Instead in the sentiment driven equilibrium the variance of the distribution of sentiments is pinned down endogenously.

\subsection{The Household}

The benchmark model features a representative household that consumes a continuum of consumption goods. Each of them is produced by a monopolistic producer indexed by $j \in[0,1]$. The continuum of consumption goods $C_{j t}$ is aggregated into a "final" consumption good $C_{t}$ according to the following Dixit-Stiglitz aggregator:

$$
C_{t}=\left[\int \epsilon_{j t}^{\frac{1}{\theta}} C_{j t}^{\frac{\theta-1}{\theta}} d j\right]^{\frac{\theta}{\theta-1}},
$$

\footnotetext{
${ }^{7}$ At this point, since goods markets have not opened and goods prices have not yet been realized, there is no guarantee that labor demand should magically (automatically) equal labor supply. We will show, however, that in equilibrium, where the distribution of sentiments is pinned down, labor supply will always equal labor demand.
} 
where $\theta>1$ is the elasticity of substitution, $\epsilon_{j t}$ is a lognormally distributed iid idiosyncratic shock with unit mean. The exponential $\frac{1}{\theta}$ on the shock $\epsilon_{j t}$ is a normalization device to simplify expressions later on. ${ }^{8}$ The representative household derives utility from aggregate consumption $C_{t}$ and leisure $1-N_{t}$ according to the utility function,

$$
\log C_{t}+\psi\left(1-N_{t}\right)
$$

subject to the budget constraint

$$
\int_{0}^{1} P_{j t} C_{j t} d j \leq W_{t} N_{t}+\Pi_{t}
$$

where $P_{j t}$ is the price of the consumption good, $N_{t}$ is aggregate labor supply, and $\Pi_{t}$ represents aggregate profit income from firms. We will normalize the competitive nominal wage $W_{t}$ to 1 . It is well known that the budget constraint (3) can be simplified to

$$
P_{t} C_{t} \leq W_{t} N_{t}+\Pi_{t}
$$

by defining $P_{t}$ as the aggregate price associated with the Dixit-Stiglitz function:

$$
P_{t}=\left[\int_{0}^{1} \epsilon_{j t}\left(P_{j t}\right)^{1-\theta} d j\right]^{\frac{1}{1-\theta}} .
$$

Given any consumption level $C_{t}$, the households' optimal consumption demand on each good is then given by

$$
C_{j t}=\left(\frac{P_{t}}{P_{j t}}\right)^{\theta} \epsilon_{j t} C_{t}
$$

Denote $Y_{t}$ as aggregate output, $Y_{j t}$ as the production of firm $j$ and $N_{j t}$ as its labor input. Then a firm $j^{\prime} s$ profit is given by $\Pi_{j t}=P_{j t} Y_{j t}-W_{t} N_{j t}$ and the aggregate profit $\Pi_{t}=\int_{0}^{1} \Pi_{j t} d j$. Hence, the total nominal GDP is given by $\int_{0}^{1} P_{j t} Y_{j t} d j$. Then

$$
Y_{t}=\frac{1}{P_{t}} \int_{0}^{1} P_{j t} Y_{j t} d j
$$

is the real GDP or aggregate output/income in the economy. Since there are no savings, the household expects to consume all its income each period, therefore in our benchmark model the

\footnotetext{
${ }^{8}$ We may interpret $\epsilon_{j t}$ either as idiosyncratic preference shocks, or as idiosyncratic productivity shocks in the case the aggregation of intermediate goods is carried out by a final good producer.
} 
realized aggregate income equals the realized aggregate consumption if the budget constraint binds: $Y_{t}=C_{t}{ }^{9}$

Denote by $Z_{t}$ the consumer sentiments about aggregate output $Y_{t}$ at the beginning of period $t$. In other words, we treat consumer expectations of aggregate income as the source of sentiments. ${ }^{10}$ At this point production has not taken place, so the market clearing prices $P_{j t}$, the aggregate price index $P_{t}$, the real wage $\frac{1}{P_{t}}$, the profit income $\Pi_{t}$ and aggregate output $Y_{t}$ have not been realized. As a result, the actual output $Y_{t}$ and consumption $C_{t}$ are not yet observable. Based on its sentiments about aggregate output, the household believes that aggregate consumption level is $C_{t}^{e}=Z_{t}$, and aggregate price is $P_{t}^{e}$ and the expected profit is $\Pi_{t}^{e}$, which all depend on the anticipated aggregate output level $Z_{t}$. Choosing labor supply to maximize utility, given expected aggregate consumption $C_{t}^{e}=Z_{t}$ and the budget constraint (4), the first order condition yields:

$$
P_{t}^{e}=\frac{1}{\psi Z_{t}}=\frac{1}{\psi C_{t}^{e}}=\frac{1}{\psi\left[\frac{1}{P_{t}^{e}} N_{t}+\frac{\Pi_{t}^{e}}{P_{t}^{e}}\right]}
$$

Notice that the right hand side of the above equation is a decreasing function of aggregate labor supply $N_{t}$, given $\frac{1}{P_{t}^{e}}$ and $\frac{\Pi_{t}^{e}}{P_{t}^{e}}$. Hence, the above equation implicitly defines labor supply as a function of sentiments: $N_{t}=N\left(Z_{t}\right)$.

\section{$2.2 \quad$ Firms}

Firms make production decisions before the goods markets open and trade takes place. Firms thus naturally try to obtain information (through market surveys or forecasting agencies or early sales) about the specific demand $C_{j t}$ for their products and the associated aggregate demand $C_{t}$ before production and hiring decisions. ${ }^{11}$ They face a nominal wage $W_{t}=1$, and a downward sloping demand curve given by (6), but prices, the real wage and aggregate output have not yet been realized. We assume therefore that firms make optimal employment and production decisions on the basis of market signals about household sentiments $Z_{t}$ and idiosyncratic demand shocks $\varepsilon_{j t}$. In particular, as in the Lucas island model, we assume that firms receive a noisy signal $s_{j t}$ that is a weighted average of firm-level demand $\epsilon_{j t}$ and the expected aggregate demand of households:

$$
s_{j t}=\lambda \log \epsilon_{j t}+(1-\lambda) \log Z_{t}+v_{j t}
$$

\footnotetext{
${ }^{9}$ Formally $W_{t} N_{t}+\Pi_{t}=W_{t} N_{t}+\int_{0}^{1}\left(P_{j t} Y_{j t}-W_{t} N_{j t}\right) d j=P_{t} Y_{t}$, and by the budget constraint we have $C_{t}=Y_{t}$.

${ }^{10}$ Our model can be generalized to have heterogeneous households with idiosyncratic but correlated sentiments. See Benhabib, Wang and Wen (2013).

${ }^{11}$ For example, one source of such information is the Michigan Consumer Surveys, which contain information about sampled consumer sentiments and other information about expected consumer demand.
} 
where $\lambda \in[0,1]$ is the weight parameter and $v_{j t}$ is an idiosyncratic noise that further contaminates the signal. ${ }^{12}$ The firms therefore face a signal extraction problem even if the variance of $v_{j t}$ is zero $\left(\sigma_{v}^{2}=0\right)$ because uncertainty about the aggregate and idiosyncratic components of demand is not resolved until outputs are sold and markets are cleared by equilibrium prices. Various possible microfoundations for how the signal is precisely generated is discussed and analyzed in section 3, where we provide explicit microfoundations that endogenize the value of $\lambda$. For now we treat $\lambda$ as a parameter.

On the basis of the signal, each firm chooses its employment and production to maximize expected profits. The intermediate goods production function is given by

$$
Y_{j t}=A N_{j t}
$$

and the nominal profit function is $\Pi_{j t}=P_{j t} Y_{j t}-\frac{W_{t}}{A} Y_{j t}$. Substituting the demand function in equation (6) into the firm's profit maximizing program we have:

$$
\max _{Y_{j t}} E\left[\left(P_{t} Y_{j t}^{1-\frac{1}{\theta}}\left(\epsilon_{j t} C_{t}\right)^{\frac{1}{\theta}}-\frac{1}{A} Y_{j t}\right) \mid s_{j t}\right] .
$$

At this point aggregate output has not yet been realized. The firms, however, believe that aggregate demand will equal aggregate supply, which in turn will equal consumer's expected aggregate income, namely, $Y_{t}=C_{t}=C_{t}^{e}=Z_{t}$, and that the aggregate price $P_{t}$ is given by $P_{t}^{e}=\frac{1}{\psi Z_{t}}$. Setting $C_{t}=Z_{t}$ in the optimization problem (11), the first order condition for the optimal supply $Y_{j t}$ is given by

$$
\left(1-\frac{1}{\theta}\right) Y_{j t}^{-\frac{1}{\theta}} E\left[P_{t}\left(\epsilon_{j t} Z_{t}\right)^{\frac{1}{\theta}} \mid s_{j t}\right]=\frac{1}{A}
$$

Substituting for $P_{t}$ in the above equation using equation (8) gives

$$
Y_{j t}=\left\{\left(1-\frac{1}{\theta}\right) \frac{A}{\psi} E\left[\left(\epsilon_{j t}\right)^{\frac{1}{\theta}} Z_{t}^{\frac{1}{\theta}-1} \mid s_{j t}\right]\right\}^{\theta} .
$$

The firm's labor demand is then simply given by $N_{j t}=Y_{j t} / A \cdot{ }^{13}$ Note also that integrating profits over firms, the aggregate profits $\Pi_{t}$ will depend only on $Z_{t}$ and not on the idiosyncratic shocks.

\footnotetext{
${ }^{12}$ The idiosyncratic noise $v_{j t}$ in the signal can also arise for example as a sampling error if firms survey a finite subset of consumers with each receiving heterogenous but correlated sentiments, as analyzed in Benhabib, Wang and Wen (2013). However introducing $v_{j t}$ is unnecessary for our basic results that follow. To eliminate its effect, we can simply set $\sigma_{v}^{2}=0$ in all Propositions that follow without loss of generality.

${ }^{13}$ We can also replace the firm's problem with $\max _{Y_{j t}} E\left[\Lambda_{t}\left(P_{t} Y_{j t}^{1-\frac{1}{\theta}}\left(\epsilon_{j t} C_{t}\right)^{\frac{1}{\theta}}-\frac{W_{t}}{A} Y_{j t}\right) \mid s_{j t}\right]$, where $\Lambda_{t}$ is the marginal utility of the household. The presence of $\Lambda_{t}$ does not matter because, with the nominal wage normalization $W=1$, the value of $\Lambda_{t}$ is constant.
} 
It is important to note from (13) that, since $\frac{1}{\theta}-1<0$, the optimal firm output declines with aggregate demand if we ignore the signal extraction problem. This implies that we have strategic substitutability across firms' actions (without informational frictions). Despite this, we will show that the equilibrium is not unique because informational frictions can create an informational strategic complementarity that gives rise to multiple sentiment-driven equilibria - a contribution of this paper.

After goods are produced, they are taken to markets and market-clearing prices are then realized. Notice that once goods are produced, their supply is fixed or predetermined by production. Exchanges in the goods markets will then determine the market clearing prices according to the demand curves of the household.

\subsection{Rational Expectations Equilibrium (REE)}

Definition 1 An REE is a sequence of allocations $\left\{C\left(Z_{t}\right), Y\left(Z_{t}\right), C_{j}\left(Z_{t}, \epsilon_{j t}\right), Y_{j}\left(Z_{t}, \epsilon_{j t}\right), N\left(Z_{t}\right)\right.$, $\left.N_{j}\left(Z_{t}, \epsilon_{j t}\right), \Pi\left(Z_{t}\right)\right\}$, prices $\left\{P\left(Z_{t}\right), P_{j}\left(Z_{t}, \epsilon_{j t}\right), W_{t}=1\right\}$, and a distribution of $Z_{t}, \mathbf{F}\left(Z_{t}\right)$, such that for each realization of $Z_{t}$, (i) equations (6) and (8) maximize household utility given the equilibrium prices $P_{t}=P\left(Z_{t}\right), P_{j t}=P_{j}\left(Z_{t}, \epsilon_{j t}\right)$ and $W_{t}=1$; (ii) equation (13) maximizes intermediategood firm's expected profits for all $j$ given the equilibrium prices $P\left(Z_{t}\right)$ and $W_{t}=1$, and the signal in equation (9); (iii) all markets clear: $C_{j t}=Y_{j t}, N\left(Z_{t}\right)=\int N_{j t} d j$, and (iv) beliefs are rational such that $P_{t}^{e}=P\left(Z_{t}\right), \Pi_{t}^{e}=\Pi\left(Z_{t}\right)$ and in particular $Z_{t}=Y_{t}$, namely, the actual aggregate output $Y_{t}$ follows a distribution consistent with $\mathbf{F}: \operatorname{Pr}\left(Y_{t} \leq X_{t}\right)=\mathbf{F}\left(X_{t}\right)$.

In equilibrium the aggregate consumption function (1), the optimal intermediate-good supply (13), and the signal (9) under the correct belief can be rewritten, respectively, as a 4-equation system:

$$
\begin{gathered}
Y_{t}=\left[\int \epsilon_{j t}^{\frac{1}{\theta}} Y_{j t}^{\frac{\theta-1}{\theta}} d j\right]^{\frac{\theta}{\theta-1}} \\
Y_{j t}=\left\{\left(1-\frac{1}{\theta}\right) \frac{A}{\psi} E\left[\left(\epsilon_{j t}\right)^{\frac{1}{\theta}} Z_{t}^{\frac{1}{\theta}-1} \mid s_{j t}\right]\right\}^{\theta} \\
s_{j t}=\lambda \log \epsilon_{j t}+(1-\lambda) \log Z_{t}+v_{j t} \\
Z_{t}=Y_{t},
\end{gathered}
$$

where the last equation simply states that the belief about the aggregate output/income is correct. This 4-equation system based on equations (14), (15), (16) and (17) can be used to solve for the equilibrium allocations in the benchmark model. The remaining can be determined in equilibrium 
as follows: $P_{t}=\frac{1}{\psi Y_{t}}$ by equation (8), $P_{j t}=\left(\epsilon_{j t} Y_{t}\right)^{\frac{1}{\theta}} Y_{j t}^{-\frac{1}{\theta}} P_{t}$ by equation $(6), N_{t}=\int_{0}^{1} N_{j t} d j=$ $\int_{0}^{1}\left(Y_{j t} / A\right) d j$ by the production function, and aggregate profits are $\Pi_{t}=P_{t} Y_{t}-N_{t}=\frac{1}{\psi}-N_{t}$.

\subsection{Fundamental Equilibria}

We start first by characterizing the REE under perfect information. This is the equilibrium where firms can perfectly observe their demand. In such a case the REE is characterized by a constant output $Y_{t}=Y^{*}$ and constant aggregate price level $P_{t}=P^{*}$. Under perfect information equation (15) becomes

$$
Y_{j t}^{\frac{1}{\theta}}=\left(1-\frac{1}{\theta}\right) \frac{A}{\psi} \epsilon_{j t}^{\frac{1}{\theta}} Y_{t}^{\frac{1-\theta}{\theta}}
$$

Equation (14) becomes

$$
C^{*}=Y^{*}=\frac{A}{\psi}\left(1-\frac{1}{\theta}\right)\left[\int \epsilon_{j t} d j\right]^{\frac{1}{\theta-1}}
$$

which implies

$$
P^{*}=\left(\frac{\theta}{\theta-1}\right) \frac{1}{A}\left[\int \epsilon_{j t} d j\right]^{\frac{1}{1-\theta}}
$$

If without loss of generality we normalize $\left(1-\frac{1}{\theta}\right) \frac{A}{\psi}=1$, we then have $\log Y_{t}=\frac{1}{\theta-1} \log E \exp \left(\varepsilon_{j t}\right)$, where $\varepsilon_{j t} \equiv \log \epsilon_{j t}$ has zero mean and variance $\sigma_{\varepsilon}^{2}$. Therefore, under the assumption of a lognormal distribution for $\epsilon_{j t}$ (or normal distribution for $\varepsilon_{j t}$ ),

$$
\log Y_{t}=\frac{1}{2(\theta-1)} \sigma_{\varepsilon}^{2}=\phi_{0}^{*}
$$

which is an alternative way of expressing equation (19). In this equilibrium sentiments do not matter.

We now formally characterize the fundamental REE under imperfect information in the presence of idiosyncratic noise $v_{j t}$ :

Proposition 1 Under the signal given by (9) there is a unique fundamental equilibrium characterized by constant output $\log Y_{t} \equiv y_{t}=\tilde{\phi}_{0}$ with

$$
\tilde{\phi}_{0}=\frac{1}{2}\left[\left(\frac{\theta+\theta \mu \lambda(\theta-1)+(\theta \mu \lambda(\theta-1))^{2}}{\theta^{2}(\theta-1)}\right) \sigma_{\varepsilon}^{2}+(\theta-1)(\theta \mu)^{2} \sigma_{v}^{2}\right],
$$

where $\mu=\frac{\frac{1}{\theta} \lambda \sigma_{\varepsilon}^{2}}{\sigma_{v}^{2}+\lambda^{2} \sigma_{\varepsilon}^{2}}$. 
Proof. See Appendix.

Note that $\tilde{\phi}_{0} \neq \phi_{0}^{*}$ because now the signal has the additional noise $\sigma_{v}^{2}>0$, which dilutes the information content of the signal. As $\sigma_{v}^{2} \rightarrow 0$, we get $\tilde{\phi}_{0} \rightarrow \phi_{0}^{*}$. Since in this case we still have $Y_{j t}=\epsilon_{j t} Y^{1-\theta}$, firm-level outputs depend negatively on aggregate output and this strategic substitutability implies that the fundamental equilibrium is unique.

\subsection{Sentiment-Driven Equilibria}

We now explore the existence of stochastic REE where aggregate output is not a constant but instead equal to the time-varying sentiments $Z_{t}$. We conjecture that $\log Z_{t}=\phi_{0}+z_{t}$, where $z_{t}$ is normally distributed with zero mean and variance $\sigma_{z}^{2} \cdot{ }^{14}$ Define $y_{t} \equiv \log Y_{t}-\phi_{0}$, then equation (17) becomes $y_{t}=z_{t}$.

Proposition 2 Let $\lambda \in(0,1 / 2)$, and $0 \leq \sigma_{v}^{2}<\lambda(1-2 \lambda) \sigma_{\varepsilon}^{2}$. There exists a sentiment-driven rational expectations equilibrium with stochastic aggregate output, $\log Y_{t}=y_{t}+\phi_{0}=z_{t}+\phi_{0}$, that has a mean

$$
\phi_{0}=\frac{1}{2}\left(\frac{(1-\lambda+(\theta-1) \lambda)}{\theta(1-\lambda)} \frac{1}{(\theta-1)}\right) \sigma_{\varepsilon}^{2}-\frac{(\theta-1) \sigma_{v}^{2}}{2 \theta^{2}(1-\lambda)^{2}}
$$

and a positive variance

$$
\sigma_{y}^{2}=\sigma_{z}^{2}=\frac{\lambda(1-2 \lambda)}{(1-\lambda)^{2} \theta} \sigma_{\varepsilon}^{2}-\frac{1}{(1-\lambda)^{2} \theta} \sigma_{v}^{2}
$$

Proof. See the Appendix.

To gain intuition we can ignore the noise term $v_{j t}$ in the signal for a moment. If firms believe that their signals reflect information both about changes in aggregate demand and firm-level demand shocks, then these beliefs will partially coordinate their output responses, up or down. Both the variance of the sentiment shock $\sigma_{z}^{2}$ and $\lambda$ affect the firms' optimal output responses through their signal extraction problems. Given $\lambda$ and the variance of the idiosyncratic demand shock $\sigma_{\varepsilon}^{2}$, for markets to clear for all possible realizations of the aggregate demand sentiment $z_{t}$, the variance $\sigma_{z}^{2}$ has to be precisely pinned down, as indicated in Proposition 2. The intuition for why the aggregate output expected by the household can always equal the aggregate output resulting from the optimal production decisions of all intermediate good firms for every possible realization of sentiments $Z_{t}$, is as follows. When aggregate demand is sentiment-driven, if we increase $\sigma_{z}^{2}$ the firm attributes more of the signal to an aggregate sentiment shock and, in response, reduces its

\footnotetext{
${ }^{14}$ For convenience, in the rest of the paper we denote the logarithm of the sentiment variable by $z_{t} \equiv \log Z_{t}-\phi_{0}$.
} 
output because optimal demand for intermediate goods is a downward sloping function of aggregate demand. However, the optimal supply of the firm's output depends also positively on firm-level demand shocks. If firms cannot distinguish firm-level shocks from aggregate demand shocks (as in the Lucas island model), informational strategic complementarities can arise so that higher realizations of $z$ result in higher optimal firm output for all intermediate goods firms. How strongly firm outputs respond to a sentiment shock $z$, however, depends on $\sigma_{z}^{2}$. In the sentiment-driven equilibrium, $\sigma_{z}^{2}$ is determined just at the value assuring that realized output $Y_{t}=C_{t}$ is equal to expected output $Z_{t}$ for all realizations of the sentiment. Thus the REE pins down beliefs, that is the variance of the sentiment distribution, even though sentiments are non-fundamental.

Mathematically, under the assumption that $z_{t} \sim \mathcal{N}\left(0, \sigma_{z}^{2}\right)$, the equations (14) and (15) become ${ }^{15}$

$$
y_{t} \equiv \frac{\lambda \sigma_{\varepsilon}^{2}+(1-\theta)(1-\lambda) \sigma_{z}^{2}}{\sigma_{v}^{2}+\lambda^{2} \sigma_{\varepsilon}^{2}+(1-\lambda)^{2} \sigma_{z}^{2}}(1-\lambda) z_{t} .
$$

Notice the belief that $y_{t}=z_{t}$ (or $Y_{t}=Z_{t}$ ) of the household and firms in general may not be correct for arbitrary distributions of sentiments (as characterized by the variance $\sigma_{z}^{2}$ ). The belief will only be correct if $\frac{\frac{1}{\theta} \lambda \sigma_{\varepsilon}^{2}+\frac{1-\theta}{\theta}(1-\lambda) \sigma_{z}^{2}}{\sigma_{v}^{2}+\lambda^{2} \sigma_{\varepsilon}^{2}+(1-\lambda)^{2} \sigma_{z}^{2}}(1-\lambda)=1$ in equation $(25)$, or $\sigma_{z}^{2}=\frac{\lambda(1-2 \lambda)}{(1-\lambda)^{2} \theta} \sigma_{\varepsilon}^{2}-\frac{1}{(1-\lambda)^{2} \theta} \sigma_{v}^{2}$ in Proposition 2. If however $\sigma_{z}^{2} \lessgtr \frac{\lambda(1-2 \lambda)}{(1-\lambda)^{2} \theta} \sigma_{\varepsilon}^{2}-\frac{1}{(1-\lambda)^{2} \theta} \sigma_{v}^{2}$ then $y_{t} \gtrless z_{t}$, and such cases do not constitute REE.

Note also that the mean output $\phi_{0}$ in the sentiment-driven equilibrium will be lower than the output $\tilde{\phi}_{0}$ under the fundamental equilibrium, and the mean markup will be higher.

As we show in section 3 where we consider the microfoundations of the signal structures in (9) and the permissible values of $\lambda$, in some cases there may also be a continuum of equilibrium $\lambda$ values, and therefore a continuum of sentiment-driven equilibria parametrized by $\lambda$ or $\sigma_{z}^{2}$. Sentiments, realized under their equilibrium distributions parametrized by $\sigma_{z}^{2}$, serve to correlate firm decisions and give rise to a continuum of correlated equilibria.

Notice that if either $\lambda \geq \frac{1}{2}$, or $\sigma_{v}^{2}>\lambda(1-2 \lambda) \sigma_{\varepsilon}^{2}$, then equilibrium would require $\sigma_{z}^{2}<0$, suggesting that the only equilibrium is the fundamental equilibrium where $z_{t}=0$ and $y_{t}=0$. When either $\lambda \geq \frac{1}{2}$, or $\sigma_{v}^{2}>\lambda(1-2 \lambda) \sigma_{\varepsilon}^{2}$ we have $\frac{\lambda \sigma_{\varepsilon}^{2}+(1-\theta)(1-\lambda) \sigma_{z}^{2}}{\sigma_{v}^{2}+\lambda^{2} \sigma_{\varepsilon}^{2}+(1-\lambda)^{2} \sigma_{z}^{2}}(1-\lambda)<1$ for any $\sigma_{z}^{2}>0$. In this case expected output will always exceed actual output $y_{t}$, so we cannot have a rational expectations equilibrium. Hence, to have a sentiment-driven equilibrium, we require $\lambda \in\left(0, \frac{1}{2}\right)$ and $\sigma_{v}^{2}<\lambda(1-2 \lambda) \sigma_{\varepsilon}^{2}$. On the other hand, if $\lambda=0$, the signal provides no information on idiosyncratic components of demand. In this case, the fundamental equilibrium is the only equilibrium because

\footnotetext{
${ }^{15}$ To see this look at the proof of this Proposition in the Appendix.
} 
of the strategic substitutability across firms' output. So the value of $\lambda$ determines the equilibrium regimes and pins down the equilibrium value of $\sigma_{z}^{2}>0$ as a function of $\sigma_{\varepsilon}^{2}$ and $\sigma_{v}^{2}$. The extra noise $v_{j t}$ in the signal makes output in the sentiment-driven equilibrium less volatile. The reason for the smaller volatility of output when $\sigma_{v}^{2}>0$ is that the signal is more noisy, and firms attribute a smaller fraction of the signal to demand fluctuations. However, note that this requires the additional restriction that the variance of the extra noise cannot be too big, $\sigma_{v}^{2}<\lambda(1-2 \lambda) \sigma_{\varepsilon}^{2}$, to ensure that sentiments matter $\left(\sigma_{z}^{2}>0\right)$.

We can show that under the conditions in Proposition (2) the labor market clears. This is of course not surprising. Labor supply depends on the expected output (sentiments), and the labor demand depends on actual output through the production functions $N_{j t}=Y_{j t} / A$ and the aggregation under equation (14). If the beliefs are correct, in equilibrium the expected output will always equal to actual output, labor demand will always equal to labor supply. To see this, we normalize $A$ for convenience such that $\log A=\frac{1}{2} \frac{\sigma_{v}^{2}+\lambda^{2} \sigma_{\varepsilon}^{2}}{(1-\lambda)^{2}}-\frac{\theta-1}{\theta} \frac{1}{2}\left(\left(\frac{1}{\theta-1}+\frac{\lambda}{1-\lambda}\right)^{2} \sigma_{\varepsilon}^{2}+\frac{\sigma_{v}^{2}}{(1-\lambda)^{2}}\right)$. Under the belief $P_{t}=P_{t}^{e}=\frac{1}{\psi Z_{t}}$ and $\Pi_{t}=\Pi_{t}^{e}=\frac{1}{\psi}-Z_{t}$, equation (8) then defines the aggregate labor supply function $N_{t}=Z_{t}$, or $\log N_{t}=\phi_{0}+z_{t}$. The aggregate labor demand of all firms is given by:

$$
\begin{aligned}
\log \int_{0}^{1} N_{j t} d j= & \phi_{0}+\underbrace{\frac{\lambda \sigma_{\varepsilon}^{2}+(1-\theta)(1-\lambda) \sigma_{z}^{2}}{\sigma_{v}^{2}+\lambda^{2} \sigma_{\varepsilon}^{2}+(1-\lambda)^{2} \sigma_{z}^{2}}(1-\lambda) z_{t}}_{y_{t}} \\
& +\frac{\sigma_{v}^{2}+\lambda^{2} \sigma_{\varepsilon}^{2}}{2}\left[\left(\frac{\lambda \sigma_{\varepsilon}^{2}+(1-\theta)(1-\lambda) \sigma_{z}^{2}}{\sigma_{v}^{2}+\lambda^{2} \sigma_{\varepsilon}^{2}+(1-\lambda)^{2} \sigma_{z}^{2}}\right)^{2}-\frac{1}{(1-\lambda)^{2}}\right] .
\end{aligned}
$$

The labor market clearing condition is satisfied if and only if condition (24) holds, in which case the coefficient of $z_{t}$ in equation (26) is unity and the third term is zero. Note that since $Y_{t}=Z_{t}$ under labor market clearing and condition (24), the household's beliefs $P_{t}=P_{t}^{e}=\frac{1}{\psi Z_{t}}$ and $\Pi_{t}=$ $\Pi_{t}^{e}=\frac{1}{\psi}-Z_{t}$ are indeed correct in equilibrium.

\subsection{A Simple Abstract Version of the Model}

To illustrate the forces at work that produce the sentiment-driven stochastic equilibrium, we can further simplify our benchmark model and abstract from the household and the production sides. Specifically, we can ignore all constant terms and assume that the economy is log-linear around a zero steady state, ${ }^{16}$ with the optimal log-output of firms given by the following best-response

\footnotetext{
${ }^{16}$ Because the model can be solved block-recursively under the log-normality assumption, the coefficients and variances can be solved without solving for the constant terms. In fact the fixed point condition (34) is formally
} 
function:

$$
y_{j t}=E\left\{\left[\beta_{0} \varepsilon_{j t}+\beta z_{t}\right] \mid s_{j t}\right\},
$$

where again $\varepsilon_{j t}$ is an idiosyncratic demand shock and $z_{t}$ is the sentiment about the aggregate output given by:

$$
y_{t}=\int y_{j t} d j
$$

Note that ignoring constant terms we can match the best response function above to the production decisions in the benchmark model given by equation (15), simply by setting $\beta_{0}=1$ and $\beta=$ $1-\theta<0 .{ }^{17}$ However, the specification in equation (27) is more general. In principle, the coefficient $\beta$ can be either negative or positive, so we can have either strategic substitutability or strategic complementarity in firms' actions. The signal $s_{j t}$ is again given by

$$
s_{j t}=\lambda \varepsilon_{j t}+(1-\lambda) z_{t}+v_{j t}
$$

and the belief is given by

$$
z_{t}=y_{t}
$$

In the perfect information equilibrium where sentiments play no role and where firms observe their demand, equation (27) becomes $y_{j t}=\beta_{0} \varepsilon_{j t}+\beta y_{t}$, so equation (28) implies $y_{t}=\beta y_{t}$. Clearly, if $\beta \neq 1$, then the only equilibrium is a constant output $\left(y_{t}=0\right.$ or $\left.Y_{t}=Y^{*}\right)$. Note that if $\beta=1$, there is a continuum of fundamental equilibria. Under imperfect information, $y_{t}$ is also constant in the fundamental equilibrium, where equation (27) yields $y_{j t}=\beta y_{t}+\frac{\lambda \beta_{0} \sigma_{\varepsilon}^{2}}{\sigma_{v}^{2}+\lambda^{2} \sigma_{\varepsilon}^{2}}\left(v_{j t}+\lambda \varepsilon_{j t}\right)$. Substituting this solution into equation (28) and integrating again gives

$$
y_{t}=\int y_{j t} d j=\beta y_{t}
$$

So unless $\beta=1$, in which case there is a continuum of fundamental equilibria, the unique equilibrium is given by $y_{t}=0$.

In the sentiment-driven stochastic equilibrium, assume that $z_{t}$ is normally distributed with zero mean and variance $\sigma_{z}^{2}$. Based on the best response function given by equation (27), signal extraction implies

$$
y_{j t}=\frac{\lambda \beta_{0} \sigma_{\varepsilon}^{2}+(1-\lambda) \beta \sigma_{z}^{2}}{\sigma_{v}^{2}+\lambda^{2} \sigma_{\varepsilon}^{2}+(1-\lambda)^{2} \sigma_{z}^{2}}\left[v_{j t}+\lambda \varepsilon_{j t}+(1-\lambda) z_{t}\right]
$$

identical to the fixed point condition in equation (A.14) in the proof of Proposition 2 (in the appendix).

${ }^{17}$ To see this set $\beta_{0}=1$ and $\beta=1-\theta<0$ in the firm's best response function for the benchmark model, taking expectations and evaluating in log linear form in equation (A.7) in the appendix, and compare it to the log linearized best response function of the firm for this abstract model, given below by (32). 
Then, since in REE $z_{t}=y_{t}$, market clearing requires

$$
y_{t}=\int y_{j t} d j=\frac{\lambda \beta_{0} \sigma_{\varepsilon}^{2}+(1-\lambda) \beta \sigma_{z}^{2}}{\sigma_{v}^{2}+\lambda^{2} \sigma_{\varepsilon}^{2}+(1-\lambda)^{2} \sigma_{z}^{2}}(1-\lambda) y_{t} .
$$

Since this relationship has to hold for every realization of $z_{t}=y_{t}$, we need

$$
\frac{\lambda \beta_{0} \sigma_{\varepsilon}^{2}+(1-\lambda) \beta \sigma_{z}^{2}}{\sigma_{v}^{2}+\lambda^{2} \sigma_{\varepsilon}^{2}+(1-\lambda)^{2} \sigma_{z}^{2}}(1-\lambda)=1
$$

or $($ for $\beta \neq 1)$

$$
\sigma_{z}^{2}=\frac{\lambda\left(\beta_{0}-\left(1+\beta_{0}\right) \lambda\right) \sigma_{\varepsilon}^{2}-\sigma_{v}^{2}}{(1-\lambda)^{2}(1-\beta)}
$$

Thus, $\sigma_{z}^{2}$ is pinned down uniquely and it defines the sentiment-driven equilibrium. Note that if $\beta<1$, then a necessary condition for $\sigma_{z}^{2}>0$ is $\lambda \in\left(0, \frac{\beta_{0}}{1+\beta_{0}}\right)$. If $\beta_{0}=1$, this restriction becomes $\lambda \in\left(0, \frac{1}{2}\right)$, as in Proposition 2. In particular, under the usual Dixit-Stiglitz specification with strategic substitutability across intermediate goods, we have $\beta=(1-\theta)<0$. So the requirement $\beta<1$ is trivially satisfied. Note however that if $\beta>1$, which may correspond to models with externalities or increasing returns to scale, $\sigma_{z}^{2}$ will be positive if $\lambda \in\left(\frac{\beta_{0}}{1+\beta_{0}}, 1\right)$. That is, when firm output responds more than proportionately to aggregate demand $(\beta>1)$, for the sentiment-driven equilibrium to exist, the aggressive responses from firms must be moderated by the signal structure such that the signal is only weakly related to aggregate demand, that is if $\lambda \in\left(\frac{\beta_{0}}{1+\beta_{0}}, 1\right) .{ }^{18}$

\section{7 $\quad$ Stability Under Learning}

Our model is essentially static, but we can investigate whether the equilibria of the model are stable under adaptive learning. For simplicity we will confine our attention to the simplified abstract model of section (2.6), and also set $\sigma_{v}^{2}=0$, so the signal is $s_{j t}=\lambda \varepsilon_{j t}+(1-\lambda) z_{t}$. Together with this signal, REE is defined by $y_{t}=z_{t}$ and equations (27) and (28), where without loss of generality we set $\beta_{0}=1$. This model has two equilibrium solutions, the fundamental equilibrium with $\sigma_{z}^{2}=0$, and the sentiment-driven equilibrium with $\sigma_{z}^{2}=\frac{\lambda\left(\beta_{0}-\left(1+\beta_{0}\right) \lambda\right) \sigma_{\varepsilon}^{2}}{(1-\lambda)^{2}(1-\beta)}=\frac{\lambda(1-2 \lambda) \sigma_{\varepsilon}^{2}}{(1-\lambda)^{2}(1-\beta)}$. We can renormalize our model so that the sentiment shock $z_{t}$ has unit variance by redefining output as $y_{t}=\log Y_{t}=\sigma_{z} z_{t}$. The variance of output $y_{t}$ then is still $\sigma_{z}^{2}$. Solving for equilibria and rewriting equation (33) with

\footnotetext{
${ }^{18}$ In the knife-edge case where $\beta=1$, we have not only a continuum of certainty equilibria since any $y_{t}$ satisfies (31), but also a continuum of self-fulfilling stochastic equilibria since any $\sigma_{z}^{2}$ satisfies (34).
} 
$y_{t}=\sigma_{z} z_{t}$ we have:

$$
\sigma_{z} z_{t}=\frac{\lambda \sigma_{\varepsilon}^{2}+(1-\lambda) \beta \sigma_{z}^{2}}{\lambda^{2} \sigma_{\varepsilon}^{2}+(1-\lambda)^{2} \sigma_{z}^{2}}(1-\lambda) \sigma_{z} z_{t}
$$

We then obtain our previous two REEs in section (2.6): (i) $\sigma_{z}^{2}=0$ and $y_{t}=0$; and (ii) $\sigma_{z}^{2}=$ $\frac{\lambda(1-2 \lambda) \sigma_{\varepsilon}^{2}}{(1-\lambda)^{2}(1-\beta)}$ and $y_{t}=\left(\frac{\lambda(1-2 \lambda) \sigma_{\varepsilon}^{2}}{(1-\lambda)^{2}(1-\beta)}\right)^{\frac{1}{2}} z_{t}$.

We now turn to learning. Suppose that agents understand that equilibrium $y_{t}$ is proportional to $z_{t}$ and they try to learn $\sigma_{z}$. If agents conjecture at the beginning of the period $t$ that the constant of proportionality is $\sigma_{z t}=\frac{y_{t}}{z_{t}}$, then, given the optimal choices by intermediate goods firms, the realized final output is

$$
y_{t}=\frac{\lambda \sigma_{\varepsilon}^{2}+(1-\lambda) \beta \sigma_{z_{t}}^{2}}{\lambda^{2} \sigma_{\varepsilon}^{2}+(1-\lambda)^{2} \sigma_{z_{t}}^{2}}(1-\lambda) \sigma_{z t} z_{t} .
$$

Under adaptive learning with constant gains $g=1-\alpha$, agents update $\sigma_{z t}$ :

$$
\sigma_{z t+1}=\alpha \sigma_{z t}+(1-\alpha)\left(\frac{y_{t}}{z_{t}}\right)
$$

For any initial $\sigma_{z t}>0$, we will show that $\sigma_{z t}$ does not converge to 0 , which is the fundamental equilibrium. By contrast the sentiment-driven sunspot equilibrium is stable under learning provided the gain $g=1-\alpha$ is not too large.

The dynamics of $\sigma_{z t}$ are given by:

$$
\sigma_{z t+1}=\alpha \sigma_{z t}+(1-\alpha) \frac{\lambda \sigma_{\varepsilon}^{2}+(1-\lambda) \beta \sigma_{z t}^{2}}{\lambda^{2} \sigma_{\varepsilon}^{2}+(1-\lambda)^{2} \sigma_{z t}^{2}}(1-\lambda) \sigma_{z t}
$$

Let $h\left(\sigma_{z}\right)=\alpha \sigma_{z}+(1-\alpha) \frac{\lambda \sigma_{\varepsilon}^{2}+(1-\lambda) \beta \sigma_{z}^{2}}{\lambda^{2} \sigma_{\varepsilon}^{2}+(1-\lambda)^{2} \sigma_{z}^{2}}(1-\lambda) \sigma_{z}$. So we then have

$$
\sigma_{z t+1}=h\left(\sigma_{z t}\right)
$$

There are two solutions to the above fixed point problem, $\sigma_{z}=\sqrt{\frac{\lambda(1-2 \lambda) \sigma_{\varepsilon}^{2}}{(1-\lambda)^{2}(1-\beta)}}$ and $\sigma_{z}=0$. We have

$$
h^{\prime}(0)=\alpha+(1-\alpha) \frac{(1-\lambda)}{\lambda}>1
$$

as $\lambda<1 / 2$. It follows that the fundamental equilibrium $\sigma_{z}=0$ is not stable. Any initial belief of $\sigma_{z t}>0$ will lead the economy away from the fundamental equilibrium.

To check the stability of the sentiment-driven equilibrium we evaluate $h^{\prime}\left(\sigma_{z}\right)$ at $\sigma_{z}=\sqrt{\frac{\lambda(1-2 \lambda) \sigma_{\varepsilon}^{2}}{(1-\lambda)^{2}(1-\beta)}}$. This yields 


$$
h^{\prime}\left(\sigma_{z}\right)=1-(1-\alpha) 2 \sigma_{z} \frac{(1-\lambda)^{2}(1-\beta)^{2}}{\left[(1-\beta) \lambda^{2} \sigma_{\varepsilon}^{2}+\lambda(1-2 \lambda) \sigma_{\varepsilon}^{2}\right]} .
$$

So the sentiment-driven equilibrium is stable under learning if $\left|h^{\prime}\left(\sigma_{z}\right)\right|<1$. This will be true if the gain $1-\alpha$ is sufficiently small. ${ }^{19}$

\subsection{Multiple Sources of Signals}

The government and public forecasting agencies as well as news media often release their own forecasts of the aggregate economy which is influenced by consumer demand. Such public information may influence and coordinate output decisions of firms and affect the equilibria. Suppose now firms receive two signals, $s_{j t}$ and $s_{p t}$. The firm-specific signal $s_{j t}=\lambda \log \epsilon_{j t}+(1-\lambda) \log Y_{t}^{e}+v_{j t}$ is based on a firm's own preliminary information about demand, and is similarly to that in equation (9). Here $Y_{t}^{e}$ is the household's expected output level. The public signal offered by the government or news media is

$$
s_{p t}=z_{t}+e_{t}
$$

where we can interpret $e_{t}$ as common noise in the public forecast of aggregate demand with mean 0 and variance $\sigma_{e}^{2}$. For example, if consumer sentiments were heterogeneous and differed by iid shocks, then a survey of a subset of consumer sentiments would have sampling noise $e_{t}{ }^{20}$

We also assume that $\sigma_{e}^{2}=\tilde{\gamma} \sigma_{z}^{2}$, where $\tilde{\gamma}>0$. This assumption states that the variance of the forecast error of the public signal for aggregate demand is proportional to the variance of $z$. Then in the fundamental equilibrium where output is constant over time, the public forecast of output is correct and constant as well (i.e., $\sigma_{z}^{2}=0$ ).

Proposition 3 If $\lambda \in(0,1 / 2)$, and $\sigma_{v}^{2}<\lambda(1-2 \lambda) \sigma_{\varepsilon}^{2}$, then there exists a sentiment-driven rational expectations equilibrium with stochastic aggregate output $\log Y_{t}=y_{t}=z_{t}+\eta e_{t}+\phi_{0} \equiv \hat{z}_{t}+\phi_{0}$, which has mean $\phi_{0}=\frac{1}{2}\left(\frac{(1-\lambda+(\theta-1) \lambda)}{\theta(1-\lambda)} \frac{1}{(\theta-1)}\right) \sigma_{\varepsilon}^{2}-\frac{(\theta-1) \sigma_{v}^{2}}{2 \theta^{2}(1-\lambda)^{2}}$ and variance $\sigma_{y}^{2}=\sigma_{\tilde{z}}^{2}=\frac{\lambda(1-2 \lambda)}{(1-\lambda)^{2} \theta} \sigma_{\varepsilon}^{2}-$ $\frac{1}{(1-\lambda)^{2} \theta} \sigma_{v}^{2}>0$ with $\eta=-\frac{\sigma_{z}^{2}}{\sigma_{e}^{2}}=-\frac{1}{\tilde{\gamma}}$. In addition, there is an equilibrium with constant output identical to that given in Proposition 1 with $\sigma_{z}^{2}=\tilde{\gamma} \sigma_{e}^{2}=0$.

Proof. See the Appendix.

As shown in the proof of Proposition 3, firms choose their optimal output based both on $z_{t}$ and $e_{t}$. When $\sigma_{\hat{z}}^{2}=\frac{\lambda(1-2 \lambda)}{(1-\lambda)^{2} \theta} \sigma_{\varepsilon}^{2}-\frac{1}{(1-\lambda)^{2} \theta} \sigma_{v}^{2}$, the optimal weight that they place on the public

\footnotetext{
${ }^{19}$ We thank George Evans, Bruce McGough and Ramon Marimon for pointing out that the stability of the sentimentdriven equilibria also easily obtains under the simpler learning rules, based for example on simple forecasts of $\sigma_{z t}$ based averaging of its past values.

${ }^{20}$ See Benhabib, Wang and Wen (2013).
} 
signal becomes zero. Nevertheless aggregate output is stochastic, and driven by the volatility of $\hat{z}_{t} \equiv z_{t}+\eta e_{t}$. It is easy to see that the equilibrium of Proposition 1 with $\sigma_{z}^{2}=0$ also applies to Proposition 3 since we have $\sigma_{e}^{2}=\tilde{\gamma} \sigma_{z}^{2}=0$, i.e. the public signal also becomes a constant. We can then directly apply Proposition 1 to find the equilibrium output (see the proof in Appendix).

As in the one signal case, the signal extraction problem delivers the optimal weights for the firm-specific and public signals, $\xi_{0}$ and $\xi_{1}$, respectively. The first order conditions of the firm relate the optimal output of each firm,

$$
y_{j t} \equiv(1-\theta) \phi_{0}+\theta \log E\left[\exp \left(\frac{1}{\theta} \varepsilon_{j t}+\frac{1-\theta}{\theta}\left(z_{t}+\eta e_{t}\right)\right) \mid\left\{s_{j t}, s_{p t}\right\}\right]
$$

to their signals $\left\{s_{j t}, s_{p t}\right\}$ in terms of the parameters of the model (see equation (A.31) in the appendix). Then, again as in the one signal case, we can solve for the variance $\sigma_{z}^{2}$ in terms of model parameters as well as $\xi_{0}$ and $\xi_{1}$, such that for every realization of the sentiment $z_{t}$ aggregate output $y_{t}$ is equal to the output expected by households $y_{t}=z_{t}$, based on their sentiments. These conditions, for the optimality of weights and for market clearing, restrict the weights on the two signals, $\xi_{0}, \xi_{1}$, as well as the variance of sentiments $\sigma_{z}^{2}$, as in the one signal case. However, we now have one additional degree of freedom, $\eta$, introduced in the solution for aggregate output, which relates $y_{t}$ to the noise on the public signal $e_{t}$. We can now choose $\eta$ so that at the optimal solution the weights are $\xi_{1}=0$ and $\xi_{0}>0$. They satisfy the optimality conditions for the choice of weights, as well as the aggregate market clearing conditions. But $\xi_{1}=0$ implies a zero weight for the public signal. Intuitively, the reason is that given the particular choice of $\eta$, the covariance between the quantity $x_{j t}=\varepsilon_{j t}+(1-\theta)\left(z_{t}+\eta e_{t}\right)$ optimally targeted by firm $j$ and the public signal, as well as the correlation between the firm-specific signal $s_{j t}$ and the public signal $s_{p t}$, become exactly zero, that is, $E\left(x_{j t} s_{p t}\right)=\operatorname{cov}\left(s_{j t}, s_{p t}\right)=0$. Therefore, while all optimality and market clearing conditions are satisfied, the particular choice of $\eta$ allows us to construct a sentiment-driven equilibrium where the weight on the public signal is exactly equal to zero. Nonetheless, the public signal is not irrelevant. The noise on the public signal, $e_{t}$, is already incorporated into aggregate output $y_{t}$ with weight $\eta$, but once this noise is incorporated into $y_{t}$, then the public signal gets zero weight for determining optimal output.

\section{Microfoundations for the Signals}

Since intermediate goods firms make employment and production decisions prior to the realization of market clearing prices, they have to form expectations about their demand and the real wage. So far we simply assumed that firms receive signals of the type given in equation (9) based on their market research, market surveys, early orders, initial inquiries and advanced sales, to form 
such expectations. In particular, we assumed that the signals obtained by intermediate goods firms consist of a weighted sum of the fundamental shock to firm-level demand and the sentiment shock to aggregate demand, and that the relative weights $\lambda$ and $(1-\lambda)$ attached to these shocks are exogenous. It is therefore desirable to spell out in more detail the microfoundations of how firms obtain these noisy signals and how the weights are determined in the signals. in the signals

We begin with a special signal structure that reveals, up to any iid noises, the "correct" weights of the fundamental shock and the sentiment shock that the intermediate goods firms "want" to know in order to make their optimal production and employment decisions. We show that such a signal, under the simple informational structures of our model, would eliminate the signal extraction problem of the firm and exclude the possibility of sentiment-driven equilibria. ${ }^{21}$

To see this, note that the demand curve of firm $j$ is given by $Y_{j t}=\left(\frac{P_{j t}}{P_{t}}\right)^{-\theta} Z_{t} \epsilon_{j t}$. Since from the household first-order conditions we have $P_{t}=\frac{1}{\psi Z_{t}}$, the logarithm of the demand curve, ignoring constants that can be filtered, becomes $y_{j t}=\varepsilon_{j t}+(1-\theta) z_{t}-\theta p_{j t}$. Suppose firm $j$ can post a hypothetical price $\tilde{p}_{j t}$ and ask a subset of consumers about their intended demand given this hypothetical price. The firms can then obtain a signal about the intercept of their demand curve, $s_{j t}=\varepsilon_{j t}+(1-\theta) z_{t}+v_{j t}$, possibly with some noise $v_{j t}$ if consumers have heterogenous preferences or sentiments. ${ }^{22}$ The optimal output decision of firms, under their belief that $y_{t}=z_{t}$, is then given by

$$
y_{j t}=E\left[\varepsilon_{j t}+(1-\theta) y_{t}\right] \mid s_{j t}=\frac{\sigma_{s}^{2}-\sigma_{v}^{2}}{\sigma_{s}^{2}}\left[\varepsilon_{j t}+(1-\theta) y_{t}+v_{j t}\right]
$$

where $\sigma_{s}^{2}$ is the variance of the signal. ${ }^{23}$ Integrating across firms based on $y_{t}=\int y_{j t} d j$, and equating coefficients of $y_{t}$ yields $1=\frac{\sigma_{s}^{2}-\sigma_{v}^{2}}{\sigma_{s}^{2}}(1-\theta)$. This equality is impossible (even if $\sigma_{v}^{2}=0$ ) since by construction $\sigma_{s}^{2}>\sigma_{v}^{2}$ and $\theta>1 .^{24}$ In other words, a constant output with $y_{t}=0$ is the only equilibrium. The intuition is that under such special signal structures the firms can perfectly filter out the noises and obtain "correct" information about the "true" demand of their goods in equilibrium.

To restore the possibility of sentiment-driven equilibria, we can either slightly complicate the signal extraction problem of the firm by adding an extra source of uncertainty, or we can modify

\footnotetext{
${ }^{21}$ We thank an anonymous referee for pointing this out to us.

${ }^{22}$ See Benhabib, Wang and Wen (2013) for the case where consumer sentiments are heterogenous but correlated.

${ }^{23}$ Note that $\sigma_{s}^{2}-\sigma_{v}^{2}>0$ is the variance of $\varepsilon_{j t}+(1-\theta) y_{t}$, or the covariance $\operatorname{Cov}\left(\varepsilon_{j t}+(1-\theta) y, s_{j t}\right)$.

${ }^{24}$ In general, a model with the following signal extraction problem, $y_{j t}=E\left[\beta_{0} \varepsilon_{j t}+\beta y_{t}\right] \mid\left[\beta_{0} \varepsilon_{j t}+\beta y_{t}+v_{j t}\right]$ and the aggregation $y_{t}=\int y_{j t} d j$ cannot have sentiment-driven equilibria. To see that, we note $y_{j t}=$ $\frac{\beta_{0}^{2} \sigma_{\varepsilon}^{2}+\beta^{2} \sigma_{z}^{2}}{\beta_{0}^{2} \sigma_{\varepsilon}^{2}+\beta^{2} \sigma_{z}^{2}+\sigma_{v}^{2}}\left[\beta_{0} \varepsilon_{j t}+\beta y_{t}+v_{j t}\right]$. A sentiment equilibrium requires $1=\frac{\beta_{0}^{2} \sigma_{\varepsilon}^{2}+\beta^{2} \sigma_{z}^{2}}{\beta_{0}^{2} \sigma_{\varepsilon}^{2}+\beta^{2} \sigma_{z}^{2}+\sigma_{v}^{2}} \beta$, which is impossible since $\beta<1$ and $0<\frac{\beta_{0}^{2} \sigma_{\varepsilon}^{2}+\beta^{2} \sigma_{z}^{2}}{\beta_{0}^{2} \sigma_{\varepsilon}^{2}+\beta^{2} \sigma_{z}^{2}+\sigma_{v}^{2}}<1$.
} 
the signal so that it does not eliminate the signal extraction problem faced by the firm. We provide microfoundations for both of these approaches below.

First we study a model with an additional source of uncertainty. We still allow a firm to post a hypothetical price $\tilde{p}_{j t}$ and to ask a subset of consumers about their intended demand at that hypothetical price. However, at the time of the survey the preference shock is not yet realized with certainty: each consumer $i$ receives a signal for his/her goods-specific preference shock $\epsilon_{j t}$ : $s_{h t}^{j}=\varepsilon_{j t}+h_{j t}^{i}$, which forms the basis of their response to the posted hypothetical price. This extra source of uncertainty now enters the demand signal received by the intermediate goods firms, and re-establishes the sentiment-driven equilibria.

In our second approach the firms, instead of learning the demand for their good for a particular hypothetical posted price, receive a signal from consumers about the quantity of the demand for their good. Consumers respond to demand surveys on the basis of their expectations of equilibrium prices. Firms therefore still face downward sloping demand curves, but the signal transmitted to them is now only a quantity signal. Therefore they still have to optimally extract from their signal the magnitude of the fundamental and sentiment shocks because the realization of prices and real wages depend on the relative magnitude of these shocks. We show that (i) a continuum of endogenous sentiment-driven equilibria arise in this setup even if firms can observe the quantity of their demand perfectly (i.e., even if their signal is $s_{j t}=y_{j t}$ ) $;^{25}$ and (ii) the signal $s_{j t}=y_{j t}$ is isomorphic to that specified in equation (9) with $\lambda \in\left(0, \frac{1}{2}\right)$.

Finally we construct another case where we introduce extra uncertainty on the firm's cost side, where a cost shock is correlated with the preference or intermediate-good demand shock, possibly because a high demand may affect marketing or sales costs for the firm.

\subsection{Consumer Uncertainty}

To re-establish sentiment-driven equilibria when firms can extract information about the intercept of their demand curves by posting hypothetical prices, we introduce an additional informational friction into the benchmark model. Consider the aggregate utility function, $\frac{C_{t}^{1-\gamma}-1}{1-\gamma}-\psi N_{t}$, or alternatively the utility function, $\log \left(C_{t}-\psi \frac{N_{t}^{1+\gamma}}{1+\gamma}\right)$. Both utility functions yield, using the first order conditions in the labor market, the same first order conditions, $p_{t} \equiv-\gamma z_{t}$, where $\gamma \neq 1(\gamma=1$ corresponds to our benchmark model). There are a continuum of identical consumers indexed by $i \in[0,1]$. Now suppose each firm $j$ conducts early market surveys by posting a hypothetical price $p_{j t}$ to consumer $i$. As before, consumers' demand for good $j$ can be affected both by the aggregate sentiment $Z_{t}$ and the variety-specific preference shock $\epsilon_{j t}$, where $Z_{t}=Y_{t}$ in REE. However, we

\footnotetext{
${ }^{25}$ We keep the notation that lower-case letters denote the demeaned logarithm of the capital-letter variables.
} 
assume that at the moment of the survey, the preference shock $\epsilon_{j t}$ is not yet established with certainty, but that each consumer $i$ receives a signal for his/her preference shock $\epsilon_{j t}: s_{h t}^{j}=\varepsilon_{j t}+h_{j t}^{i}{ }^{26}$ So if firm $j$ posts a hypothetical price $\tilde{P}_{j t}$ to consumer $i$, the demand for variety $j$ at the posted price $\tilde{P}_{j t}$ will be

$$
\tilde{Y}_{j t}^{i}=\left(\frac{\tilde{P}_{j t}}{P_{t}}\right)^{-\theta} Z_{t}\left(E\left[\exp \left(\frac{1}{\theta} \varepsilon_{j t}\right) \mid\left(\varepsilon_{j t}+h_{j t}^{i}\right)\right]\right)^{\theta} .
$$

Notice that all consumers $i \in[0,1]$ are identical up to their idiosyncratic signal $h_{j t}^{i}$. Aggregating across the consumers yields

$$
\tilde{Y}_{j t}=\left(\frac{\tilde{P}_{j t}}{P_{t}}\right)^{-\theta} Z_{t} \int_{0}^{1}\left(E\left[\exp \left(\frac{1}{\theta} \varepsilon_{j t}\right) \mid\left(\varepsilon_{j t}+h_{j t}^{i}\right)\right]\right)^{\theta} d i
$$

Using the first order condition $p_{t}=-\gamma z_{t}$, the intercept of the demand curve for variety $j$ (in logarithms) is given by

$$
(1-\theta \gamma) z_{t}+\int_{0}^{1} E\left[\varepsilon_{j t} \mid\left(\varepsilon_{j t}+h_{j t}^{i}\right)\right] d j=\frac{\sigma_{\varepsilon}^{2}}{\sigma_{\varepsilon}^{2}+\sigma_{h}^{2}} \varepsilon_{j t}+(1-\theta \gamma) z_{t}
$$

Hence, the signal that firm $j$ can obtain through its market surveys is $s_{j t}=\frac{\sigma_{\varepsilon}^{2}}{\sigma_{\varepsilon}^{2}+\sigma_{h}^{2}} \varepsilon_{j t}+(1-\theta \gamma) z_{t}$, which is isomorphic to

$$
s_{j t}=\frac{\frac{\sigma_{\varepsilon}^{2}}{\sigma_{\varepsilon}^{2}+\sigma_{h}^{2}}}{\frac{\sigma_{\varepsilon}^{2}}{\sigma_{\varepsilon}^{2}+\sigma_{h}^{2}}+(1-\theta \gamma)} \varepsilon_{j t}+\left[1-\frac{\frac{\sigma_{\varepsilon}^{2}}{\sigma_{\varepsilon}^{2}+\sigma_{h}^{2}}}{\frac{\sigma_{\varepsilon}^{2}}{\sigma_{\varepsilon}^{2}+\sigma_{h}^{2}}+(1-\theta \gamma)}\right] z_{t} .
$$

Clearly, for sentiment-driven equilibria to exist, as required by the Propositions of section 2.5, we need

$$
\lambda \equiv \frac{\frac{\sigma_{\varepsilon}^{2}}{\sigma_{\varepsilon}^{2}+\sigma_{h}^{2}}}{\frac{\sigma_{\varepsilon}^{2}}{\sigma_{\varepsilon}^{2}+\sigma_{h}^{2}}+(1-\theta \gamma)} \in\left(0, \frac{1}{2}\right)
$$

which will hold if $1-\theta \gamma>\frac{\sigma_{\varepsilon}^{2}}{\sigma_{\varepsilon}^{2}+\sigma_{h}^{2}} \cdot{ }^{27}$

\footnotetext{
${ }^{26}$ The idiosyncratic consumer uncertainty about their preference shock introduced here is related to the case where consumers receive heterogenous but correlated sentiments, considered in detail in Benhabib, Wang and Yi (2013).

${ }^{27}$ This restriction on the value of $\gamma$ can be further relaxed if we extend our model to allow for heterogenous labor supply so that each intermediate goods firm faces its own wage rate.
} 


\subsection{Quantity Signal}

Instead of introducing additional sources of uncertainty to recapture sentiment-driven equilibria, we suppose the intermediate-good firms, instead of learning the demand schedule for their good for a particular hypothetical posted price, receive a signal from consumers about the quantity of the demand $C_{j t}$ for their good. We assume that the representative household's utility is $\log C_{t}-\psi N_{t}$. The household demand function for each variety is:

$$
C_{j t}=\left(\frac{P_{t}}{P_{j t}}\right)^{\theta} \epsilon_{j t} Z_{t}=\left(\frac{P_{t}}{P_{j t}}\right)^{\theta} \epsilon_{j t} Z_{t} .
$$

The first-order condition for labor supply, as in the benchmark model, yields $P_{t}=\frac{1}{\psi} \frac{1}{Z_{t}}$, with the nominal wage normalization $W_{t}=1$.

The intermediate-good firms, based on the quantity signal $s_{j t}=c_{j t}$ and the belief that $Y_{t}=Z_{t}$, choose their production according to the first-order condition

$$
Y_{j t}=\left\{\left(1-\frac{1}{\theta}\right) \frac{A}{\psi} E_{t}\left[\epsilon_{j t}^{\frac{1}{\theta}} Z_{t}^{\frac{1}{\theta}-1} \mid S_{j t}\right]\right\}^{\theta}
$$

We conjecture that in equilibrium,

$$
c_{j t}=y_{j t}=\phi \varepsilon_{j t}+\phi_{z} z_{t}
$$

where $\phi$ and $\phi_{z}$ are undetermined coefficients. Under the assumption of lognormal distributions, our model is log-linear. Defining $\beta \equiv 1-\theta$ and log-linearizing equation (52) yield

$$
\begin{aligned}
y_{j t} & =E_{t}\left[\left(\varepsilon_{j t}+\beta Z_{t}\right) \mid c_{j t}\right] \\
& =E_{t}\left[\left(\varepsilon_{j t}+\beta Z_{t}\right) \mid\left(\phi \varepsilon_{j t}+\phi_{z} z_{t}\right)\right],
\end{aligned}
$$

where $\beta \equiv 1-\theta$. By definition the aggregate output is $y_{t}=\frac{1}{\left(1-\frac{1}{\theta}\right)} \int_{0}^{1}\left(\frac{1}{\theta} \varepsilon_{j t}+\left(1-\frac{1}{\theta}\right) y_{j t}\right) d j=$ $\int_{0}^{1} y_{j t} d j$. Finally, in an REE we require consumers to have correct endogenous sentiments: for each realization of $z_{t}$,

$$
y_{t}=z_{t}
$$

Equations (53) and (54) imply

$$
\begin{aligned}
\phi \varepsilon_{j t}+\phi_{z} z_{t} & =E\left[\left(\varepsilon_{j t}+\beta z_{t}\right) \mid\left(\phi \varepsilon_{j t}+\phi_{z} z_{t}\right)\right] \\
& =\frac{\phi \sigma_{\varepsilon}^{2}+\beta \phi_{z} \sigma_{z}^{2}}{\phi^{2} \sigma_{\varepsilon}^{2}+\phi_{z}^{2} \sigma_{z}^{2}}\left(\phi \varepsilon_{j t}+\phi_{z} z_{t}\right) .
\end{aligned}
$$


Equating coefficients we have $\frac{\phi \sigma_{\varepsilon}^{2}+\beta \phi_{z} \sigma_{z}^{2}}{\phi^{2} \sigma_{\varepsilon}^{2}+\phi_{z}^{2} \sigma_{z}^{2}}=1$. Note that integrating equation (53) and using (55) we have $\phi_{z}=1$. Hence we can solve $\sigma_{z}^{2}$ as

$$
\sigma_{z}^{2}=\frac{\phi(1-\phi)}{1-\beta} \sigma_{\varepsilon}^{2}
$$

where $\beta \equiv 1-\theta<1$. So for sentiment-driven equilibria to exist with $\sigma_{z}^{2}>0, \phi$ can take any value in the interval $[0,1]$. However, the value of $\sigma_{z}^{2}$ is determined in the interval $\phi \in\left[0, \frac{1}{2}\right]$ because $\arg \max \phi(1-\phi)=\frac{1}{2}$. Therefore, since $\phi$ is indeterminate in the interval $\left[0, \frac{1}{2}\right]$, we have the following

Proposition 4 There is a continuum of sentiment-driven equilibria indexed by $\sigma_{z}^{2} \in\left[0, \frac{1}{4(1-\beta)} \sigma_{\varepsilon}^{2}\right]$.

This establishes that given the structural parameters of the model, the existence of sentimentdriven equilibria is robust to perturbations of $\sigma_{z}^{2}$ within the range $\sigma_{z}^{2} \in\left[0, \frac{1}{4(1-\beta)} \sigma_{\varepsilon}^{2}\right] .{ }^{28}$ To solve for the equilibrium prices, note that $c_{j t}$ satisfies the household's first-order conditions (51) and that $C_{t}=Z_{t}=\frac{1}{\psi} \frac{1}{P_{t}}$, which (after taking logs) can be written jointly as

$$
\begin{aligned}
c_{j t} & =\theta\left(p_{t}-p_{j t}\right)+\varepsilon_{j t}+c_{t} \\
& =-\theta p_{j t}+\varepsilon_{j t}+(1-\theta) y_{t},
\end{aligned}
$$

which can be used to solve for $p_{j t}$ and $p_{t}$.

Finally in the next proposition we show that the equilibria of this model can be mapped into the equilibria of our benchmark model parametrized by $\lambda=\frac{\phi}{\phi+1}$ :

Proposition 5 The sentiment-driven equilibria of this model with signal $s_{j t}=c_{j t}$ can be mapped one-to-one to the sentiment-driven equilibria of our benchmark model with the signal $s_{j t}=\lambda \varepsilon_{j t}+$ $(1-\lambda) z_{t}$.

Proof. First, we scale the signal by a constant, namely

$$
\phi \varepsilon_{j t}+z_{t} \Leftrightarrow \frac{\phi}{\phi+1} \varepsilon_{j t}+\frac{1}{\phi+1} y_{t}
$$

Second, define $\frac{\phi}{\phi+1}=\lambda$, so that $\phi=\frac{\lambda}{1-\lambda}$. It follows that

$$
\sigma_{z}^{2}=\frac{1}{(1-\beta)}\left(\phi-\phi^{2}\right) \sigma_{\varepsilon}^{2}=\frac{\lambda(1-2 \lambda)}{(1-\lambda)^{2} \theta} \sigma_{\varepsilon}^{2}
$$

\footnotetext{
${ }^{28}$ The results on the continuum of equilibria also hold if the signal is not on the firm specific demand $Y_{j t}$, but on aggregate demand $Y_{t}$. See Benhabib, Wang and Wen (2013).
} 
which is exactly the result of Proposition 2. Notice that $\lambda \in\left(0, \frac{1}{2}\right)$ is equivalent to $\phi \in(0,1)$.

The sentiment-driven equilibria are influenced by fundamental shocks $\varepsilon_{j t}$ and sentiments $z_{t}$, but in addition, they may also depend on firm-specific iid noise, $v_{j t}$, as shown in the following Proposition:

Proposition 6 Under the signal $s_{j t}=c_{j t}$, there also exists another type of sentiment-driven equilibria with firm-level output driven not only by the fundamental shock $\varepsilon_{j t}$ and aggregate sentiment shock $z_{t}$, but also by a firm-specific iid shock $v_{j t}$ with zero mean and variance $\sigma_{v}^{2}$ :

$$
c_{j t}=\phi \varepsilon_{j t}+z_{t}+(1+\phi) v_{j t}
$$

Furthermore the signal $s_{j t}=c_{j t}$ is isomorphic to the signal $s_{j t}=\lambda \log \epsilon_{j t}+(1-\lambda) y_{t}+v_{j t}$ in equation (9).

Proof. Given the signal $s_{j t}=c_{j t}$, conjecture that $c_{j t}=\phi \varepsilon_{j t}+z_{t}+(1+\phi) v_{j t}$. The firm's first-order condition in equation (54) becomes

$$
\begin{aligned}
\phi \varepsilon_{j t}+z_{t}+(1+\phi) v_{j t} & =E\left[\left(\varepsilon_{j t}+\beta z_{t}\right) \mid\left(\phi \varepsilon_{j t}+z_{t}+(1+\phi) v_{j t}\right)\right] \\
& =\frac{\phi \sigma_{\varepsilon}^{2}+\beta \sigma_{z}^{2}}{\phi^{2} \sigma_{\varepsilon}^{2}+\sigma_{z}^{2}+(1+\phi) \sigma_{v}^{2}}\left(\phi \varepsilon_{j t}+z_{t}+(1+\phi) v_{j t}\right) .
\end{aligned}
$$

Comparing coefficients gives $\frac{\phi \sigma_{\varepsilon}^{2}+\beta \sigma_{z}^{2}}{\phi^{2} \sigma_{\varepsilon}^{2}+\sigma_{z}^{2}+(1+\phi)^{2} \sigma_{v}^{2}}=1$, or

$$
\sigma_{z}^{2}=\frac{\phi(1-\phi) \sigma_{\varepsilon}^{2}-(1+\phi)^{2} \sigma_{v}^{2}}{(1-\beta)}
$$

Hence, there exists an continuum of sentiment-driven equilibria with $\sigma_{z}^{2}=\frac{\phi(1-\phi)-(1+\phi)^{2} \sigma_{v}^{2}}{\theta}$ and $\sigma_{v}^{2} \leq \frac{\phi(1-\phi)}{(1+\phi)^{2}} \sigma_{\varepsilon}^{2}$. Let $\lambda=\frac{\phi}{\phi+1}$ or $\phi=\frac{\lambda}{1-\lambda}$. It follows that

$$
\sigma_{z}^{2}=\frac{\lambda(1-2 \lambda) \sigma_{\varepsilon}^{2}-\sigma_{v}^{2}}{\theta(1-\lambda)^{2}}, \quad \sigma_{v}^{2} \leq \lambda(1-2 \lambda) \sigma_{\varepsilon}^{2}
$$

which exactly correspond to the results of Proposition 3. Since sunspots can exist only for $\phi \in(0,1)$, we then require $\lambda \in\left(0, \frac{1}{2}\right)$. Hence, the signal $s_{j t}=c_{j t}$ is isomorphic to the signal $s_{j t}=\lambda \log \epsilon_{j t}+$ $(1-\lambda) y_{t}+v_{j t}$ in equation (9). 


\subsection{Cost Shocks}

As in the case of consumer uncertainty of section 3.1, we assume that the household utility function is $\frac{C_{t}^{1-\gamma}-1}{1-\gamma}-\psi N_{t}$. Each firm $j$ 's total production cost (or labor productivity) is affected by an idiosyncratic shock that is correlated with the demand shock $\epsilon_{j t}$. For example, marketing costs may be lower under favorable demand conditions: for a higher amount of sales, labor becomes more productive so that labor demand $N_{j t}=Y_{j t} \epsilon_{j t}^{-\tau}$ is lower, where $\tau>0$ is a parameter. Alternatively, if marketing costs increase with sales and labor demand is higher, we may have $\tau<0$.

Firm $j$ 's problem is:

$$
\max _{Y_{j t}} E_{t}\left[\left(P_{t} Y_{j t}^{1-\frac{1}{\theta}}\left(\epsilon_{j t} Z_{t}\right)^{\frac{1}{\theta}}-\frac{W_{t}}{A} \epsilon_{j t}^{-\tau} Y_{j t}\right) \mid S_{j t}\right]
$$

Under the belief that $Y_{t}=Z_{t}$, the firm's first-order condition is

$$
Y_{j t}=\left\{\left(1-\frac{1}{\theta}\right) \frac{A}{\psi E_{t}\left[\epsilon_{j t}^{-\tau} \mid s_{j t}\right]} E_{t}\left[\epsilon_{j t}^{\frac{1}{\theta}} Z_{t}^{\frac{1}{\theta}-\gamma} \mid S_{j t}\right]\right\}^{\theta}
$$

which (after taking logs and filtering out constants) may be written as

$$
y_{j t}=E_{t}\left[\left((1+\theta \tau) \varepsilon_{j t}+(1-\theta \gamma) z_{t}\right) \mid s_{j t}\right]
$$

Now assume that firms can then obtain a signal on the intercept of their demand curve with a noise $\eta_{j t}:$

$$
s_{j t}=\varepsilon_{j t}+(1-\theta \gamma) z_{t}+\eta_{j t}
$$

Using this signal, even assuming firms can gain perfect information with $\eta_{j t} \equiv 0$, the first-order condition becomes

$$
y_{j t}=E_{t}\left[\left((1+\theta \tau) \varepsilon_{j t}+(1-\theta \gamma) z_{t}\right) \mid\left(\varepsilon_{j t}+(1-\theta \gamma) y_{t}\right)\right]
$$

Hence, we obtain

$$
y_{j t}=\frac{(1+\theta \tau) \sigma_{\varepsilon}^{2}+(1-\theta \gamma)^{2} \sigma_{z}^{2}}{\sigma_{\varepsilon}^{2}+(1-\theta \gamma)^{2} \sigma_{z}^{2}}\left(\varepsilon_{j t}+(1-\theta \gamma) z_{t}\right)
$$

Integration, since in a REE $z_{t}=y_{t}$, yields $y_{t}=\frac{(1-\theta \gamma)^{2} \sigma_{z}^{2}+(1+\theta \tau) \sigma_{\varepsilon}^{2}}{\sigma_{\varepsilon}^{2}+(1-\theta \gamma)^{2} \sigma_{z}^{2}}(1-\theta \gamma) y_{t}$, or $\frac{(1-\theta \gamma)^{2} \sigma_{z}^{2}+(1+\theta \tau) \sigma_{\varepsilon}^{2}}{\sigma_{\varepsilon}^{2}+(1-\theta \gamma)^{2} \sigma_{z}^{2}}(1-$ $\theta \gamma)=1$. Define $\beta=(1-\theta \gamma)<1$, we have

$$
\sigma_{z}^{2}=\frac{[(1+\theta \tau) \beta-1]}{\beta^{2}(1-\beta)} \sigma_{\varepsilon}^{2} .
$$


For sentiment-driven equilibria to exist we need

$$
(1+\theta \tau) \beta>1
$$

Notice that if $\tau=0$ (as in our benchmark model), sentiment-driven equilibria will not be possible, but they can exist if $\tau \neq 0$. In that case, the sign of $\tau$ depends the sign of $\beta$ : If $\beta>0$ (the case where firm output and aggregate output are complements), we need $\tau>\frac{1-\beta}{\beta \theta}$. If $\beta<0$ (the case where firm output and aggregate output are substitutes) we need $\tau<\frac{1-\beta}{\beta \theta}$ and $\tau$ can be negative.

\section{Extensions}

\subsection{Price-Setting Firms}

So far we considered cases where firms decide how much to produce before knowing their demand. We now briefly consider the case where intermediate goods firms must set prices first and commit to meeting demand at the announced prices. ${ }^{29}$ The Dixit-Stiglitz structure of our model implies that the optimal price for an intermediate goods firm under perfect information is

$$
P_{j t}=\frac{\theta}{\theta-1} W_{t}
$$

Note that whether we normalize the price of the aggregate consumption good or the nominal wage to be unity, the optimal price does not depend on idiosyncratic preference shocks. Sentiment-driven equilibria cannot exist as firms do not face signal extraction problems. Therefore we assume, as in section (3.3), that the firm's costs are positively correlated with firm's demand. We use the aggregate consumption good price as the numeraire. ${ }^{30}$ The firm's problem is

$$
\max _{Y_{j t}} E_{t}\left[\left(P_{j t}^{1-\theta} \epsilon_{j t} Z_{t}-\frac{W_{t}}{A} \epsilon_{j t}^{-\tau}\left(P_{j t}^{-\theta} \epsilon_{j t} Z_{t}\right)\right) \mid s_{j t}\right]
$$

where we substitute $N_{j t}=Y_{j t} / \epsilon_{j t}^{\tau}$ and $Y_{j t}=P_{j t}^{-\theta} \epsilon_{j t} Z_{t}$. The optimal price is then

$$
(\theta-1) P_{j t}^{-\theta} E_{t}\left[\epsilon_{j t} Z_{t} \mid S_{j t}\right]=\theta P_{j t}^{-\theta-1} E_{t}\left[W_{t} \epsilon_{j t}^{1-\tau} Z_{t} \mid S_{j t}\right]
$$

That is, $P_{j t}=\frac{\theta}{\theta-1} \frac{E_{t}\left[W_{t} \epsilon_{j t}^{1-\tau} Z_{t} \mid S_{j t}\right]}{E_{t}\left[\epsilon_{j t} Z_{t} \mid S_{j t}\right]}$. Since from the first-order condition for labor supply we have $W_{t}=\psi Z_{t}^{\gamma}$, taking logs leads to

$$
p_{j t}=E\left[\left(\gamma z_{t}-\tau \varepsilon_{j t}\right) \mid s_{j t}\right],
$$

\footnotetext{
${ }^{29}$ See the analysis in Wang and Wen (2007) for similar models. In models where money plays a role and agents choose to hold money, rigidities in price-setting can be addressed via monetary policy to alleviate or eliminate inefficient equilibria. We do not have money in our simple model of price setting, so we cannot explore the role of monetary policy.

${ }^{30}$ Our result also holds if we use the nominal wage as the numeraire.
} 
where $\varepsilon_{j t} \equiv \log \epsilon_{j t}$. The aggregate price index is normalized to unity $P_{t}=\left[\int \epsilon_{j t} P_{j t}^{1-\theta} d j\right]^{\frac{1}{1-\theta}}=1$, which implies that $\int_{0}^{1} p_{j t} d j=0$. Notice that since $s_{j t}=\lambda \varepsilon_{j t}+(1-\lambda) z_{t}$, we have

$$
p_{j t}=\frac{\gamma(1-\lambda) \sigma_{z}^{2}-\tau \sigma_{\varepsilon}^{2}}{\lambda^{2} \sigma_{\varepsilon}^{2}+(1-\lambda)^{2} \sigma_{z}^{2}}\left(\lambda \varepsilon_{j t}+(1-\lambda) z_{t}\right)
$$

Then sentiment-driven equilibria require $z_{t}=y_{t}$ and $\gamma(1-\lambda) \sigma_{z}^{2}-\tau \sigma_{\varepsilon}^{2}=0$, or

$$
\sigma_{z}^{2}=\frac{\tau \sigma_{\varepsilon}^{2}}{\gamma(1-\lambda)}>0
$$

which holds for any $\gamma$ and $\lambda$. Note that here even if firms can post a price $\tilde{P}_{j t}$ and obtain the intercept term in the demand curve $\tilde{Y}_{j t}=\tilde{P}_{j t}^{-\theta} \epsilon_{j t} Z_{t}$ which reveals the sum $\varepsilon_{j t}+y_{t}$, sentimentdriven equilibria will still exist.

\subsection{Persistence}

Persistence in output can be introduced in a variety of ways. The simplest way is to note that the productivity parameter $A$ in the benchmark model of section 2 can be a stochastic process that is observed at the beginning of each period. In this case the persistence of aggregate output would be driven by the stochastic process for $A .^{31}$

Finally, in a model with aggregate fundamental preference shocks, we may assume that the aggregate fundamental preference shock is a stochastic process but that intermediate goods firms only observe aggregate demand $C_{t}$ and its history. They do not separately observe the past or present values of the aggregate preference shocks or sentiments $Z_{t}$. Then equilibrium output will also be persistent, as shown in Benhabib, Wang and Wen (2013).

\section{Conclusion}

In their discussion of correlated sunspot equilibria, Aumann, Peck and Shell (1988) note: "Even if economic fundamentals were certain, economic outcomes would still be random... Each economic actor is uncertain about the strategies of the others. Business people, for example, are uncertain about the plans of their customers... This type of economic randomness is generated by the market economy: it is thus endogenous to the economy, but extrinsic to the economic fundamentals." Along similar lines, we explore the Keynesian idea that sentiments or animal spirits can influence the level of aggregate income and give rise to recurrent boom-bust cycles. In particular, we show

\footnotetext{
${ }^{31}$ Another possible approach to obtain persistence, making use of the multiplicity of equilibria, is to introduce a simple Markov sunspot process that selects the equilibrium in each period, alternating between the certainty and sentiment driven equilibria.
} 
that when consumption and production decisions must be made separately by consumers and firms who are uncertain of each other's plans, the equilibrium outcome can indeed be influenced by animal spirits or sentiments, even though all agents are fully rational. The key to generating our results is a natural friction in information: Even if firms can perfectly observe or forecast the demand for the goods that they produce, they cannot separately identify the components of their demand stemming from consumer sentiments (at the aggregate level) as opposed to the demand stemming from idiosyncratic preference shocks. Sentiments matter because they are correlated across consumers, and they affect aggregate demand and real wages differently than idiosyncratic preference or productivity shocks. Faced with a signal extraction problem, firms make optimal production decisions that depend on the degree of sentiment uncertainty, or the variance of the distribution of sentiment shocks. Such sentiment shocks can give rise to sentiment-driven rational expectations equilibria in addition to equilibria driven solely by fundamentals. We show that in an simple production economy, sentiments completely unrelated to fundamentals can affect output and employment even though (i) expectations are fully rational and (ii) there are no externalities, non-convexities or strategic complementarities in production. Furthermore, in our model with microfounded signals there can also exist a continuum of sentiment-driven rational expectations equilibria, parametrized by the variance of sentiment shocks. Such sentiment-driven equilibria are not based on randomizations over fundamental equilibria, and they are stable under constant gain learning if the gain parameter is not too large.

\section{References}

[1] Amador, M. and Weill, P. O. 2010. "Learning from Prices: Public Communication and Welfare", Journal of Political Economy, 866-907.

[2] Angeletos, G-M., and La'O, J., 2012. "Sentiments," Econometrica 81, 739-780.

[3] Angeletos, G-M., Lorenzoni, G. and Pavan, A. (2010). "Beauty Contests and Irrational Exuberance: A Neoclassical Approach", NBER working paper 15883.

[4] Angeletos, G-M, and La'O, J., 2009. "Noisy Business Cycles," NBER Macroeconomics Annual, 24, 319-378.

[5] Angeletos, G-M, and Pavan, A., 2007. "Efficient Use of Information and Social Value of Information," Econometrica 75, 1103-1142.

[6] Angeletos, G-M and Werning, I., 2006. "Information Aggregation, Multiplicity, and Volatility", American Economic Review, 96, 1720-1736. 
[7] Angeletos, G-M , Hellwig, C. and N. Pavan, 2006. "Signaling in a Global Game: Coordination and Policy Traps", Journal of Political Economy, 114, 452-484.

[8] Aumann, R. J., 1974. "Subjectivity and Correlation in Randomized Strategies", Journal of Mathematical Economics, 1, 67-96.

[9] Aumann, R. J.,1987. "Correlated Equilibrium as an Expression of Bayesian Rationality", Econometrica, 55, 1-18.

[10] Aumann, Robert J., Peck, James and Karl Shell,1988. "Asymmetric Information and Sunspot Equilibria: A Family of Simple Examples", CAE Working Paper \#88-34

[11] Benhabib, J. and Farmer, R., 1994, "Indeterminacy and Increasing Returns", Journal of Economic Theory 63, 19-41.

[12] Benhabib, J., Wang, P. and Wen, Y., 2013. "Uncertainty and Sentiment-Driven Equilibria", NBER working paper w18878

[13] Bergemann, D., and Morris, S., 2011. "Correlated Equilibrium in Games with Incomplete Information", Cowles Foundation Discussion Papers 1822, Cowles Foundation for Research in Economics, Yale University.

[14] Bergemann, D., Morris, S. and T. Heinmann, 2013. "Information, Interdependence, and Interaction: Where Does the Volatility Come From?", mimeo.

[15] Cass, D. and Shell, K., 1983. "Do Sunspots Matter?", Journal of Political Economy 91, 193-227.

[16] Evans, G., Honkapohja, S., 2001. Learning and Expectations in Macroeconomics. Princeton University Press.

[17] Forges, F. and Peck, J., 1995. "Correlated Equilibrium and Sunspot Equilibrium", Economic Theory, Springer, 5, 33-50.

[18] Forges, F., 2006. "Correlated Equilibrium in Games with Incomplete Information Revisited", Theory and Decision, 61: 329-344.

[19] Gaballo, G., 2012. "Private Uncertainty and Multiplicity", Banque de France, Monetary Policy Research Division, http://www.mwpweb.eu/1/98/resources/document_400_1.pdf

[20] Hart, S., and Schmeidler, D.,1989. "Existence of Correlated Equilibria." Mathematics of Operations Research, 14, 18-25. 
[21] Hellwig, C., Mukherji, A., and Tsyvinski, A., 2006. "Self-Fulfilling Currency Crises: The Role of Interest Rates", American Economic Review, 96, 1769-87.

[22] Hellwig, C. and Veldkamp L., 2009. "Knowing What Others Know: Coordination Motives in Information Acquisition", Review of Economic Studies, Wiley Blackwell, 76, 223-251.

[23] Keynes, J. M., 1936. The General Theory of Employment, Interest and Money. London. Macmillan.

[24] Lucas, R. E., Jr., 1972. "Expectations and the Neutrality of Money", Journal of Economic Theory 4, 103-124.

[25] Manzano, C. and Vives, X., 2011. "Public and Private Learning from Prices, Strategic Substitutability and Complementarity, and Equilibrium Multiplicity", Journal of Mathematical Economics, Elsevier, 47, 346-369.

[26] Maskin, E. and Tirole, J., 1987, "Correlated Equilibria and Sunspots", Journal of Economic Theory 43, 364-373.

[27] Morris, S. and Shin, H. S., 1998. "Unique Equilibrium in a Model of Self-Fulfilling Currency Attacks", American Economic Review, 88, 587-597.

[28] Morris, M., and Shin, H. S., 2002. "The Social Value of Public Information," American Economic Review, 92, 1521-1534/

[29] Peck, J. and Shell, K., 1991. "Market Uncertainty: Correlated and Sunspot Equilibria in Imperfectly Competitive Economies", Review of Economic Studies, 58, 1011-29.

[30] Spear, S. E., 1989. "Are Sunspots Necessary?", Journal of Political Economy, 97, 965-973

[31] Stein, N. D., Parrilo, P. A., and Ozdaglar, A., 2011. "Correlated Equilibria in Continuous Games: Characterization and Computation." Games and Economic Behavior, 71, 436-455

[32] Wang, P. and Wen, Y., 2007. "Incomplete Information and Self-fulfilling Prophecies", Working Papers 2007-033, Federal Reserve Bank of St. Louis, Revised, 2009. 


\section{A Appendix}

This Appendix provides brief proofs for Propositions 1-3 in the paper. More detailed proofs can be found in our NBER working paper (Benhabib, Wang and Wen, 2012).

\section{A. 1 Proofs of Proposition 1 and Proposition 2}

Proof. We start with the proof of Proposition 2, and give the proof of Proposition 1 further below.

1. The Sentiment-Driven Equilibrium. Let $s_{j t}=v_{j t}+\lambda \varepsilon_{j t}+(1-\lambda) z_{t}$. Firms conjecture that output is equal to

$$
\log Y_{t}=y_{t}=\phi_{0}+z_{t}
$$

where $\phi_{0}$, and $\sigma_{z}^{2}$ are constants to be determined. The optimal output of a firm can be written as

$$
y_{j t}=(1-\theta) \phi_{0}+\theta \log E_{t}\left[\exp \left(\frac{1}{\theta} \varepsilon_{j t}+\frac{1-\theta}{\theta} z_{t}\right) \mid s_{j t}\right]
$$

Note that

$E_{t}\left[\exp \left(\frac{1}{\theta} \varepsilon_{j t}+\frac{1-\theta}{\theta} z_{t}\right) \mid s_{j t}\right]=\exp \left(E\left[\left(\frac{1}{\theta} \varepsilon_{j t}+\frac{1-\theta}{\theta} z_{t}\right) \mid s_{j t}\right]+\frac{1}{2} \operatorname{var}\left(\left(\frac{1}{\theta} \varepsilon_{j t}+\frac{1-\theta}{\theta} z_{t}\right) \mid s_{j t}\right)\right)$

where

$$
\begin{aligned}
E\left[\left(\frac{1}{\theta} \varepsilon_{j t}+\frac{1-\theta}{\theta} z_{t}\right) \mid s_{j t}\right] & =\frac{\operatorname{cov}\left(\frac{1}{\theta} \varepsilon_{j t}+\frac{1-\theta}{\theta} z_{t}, s_{j t}\right)}{\operatorname{var}\left(s_{j t}\right)} s_{j t} \\
& =\frac{\frac{1}{\theta} \lambda \sigma_{\varepsilon}^{2}+\frac{1-\theta}{\theta}(1-\lambda) \sigma_{z}^{2}}{\sigma_{v}^{2}+\lambda^{2} \sigma_{\varepsilon}^{2}+(1-\lambda)^{2} \sigma_{z}^{2}}\left(v_{j t}+\lambda \varepsilon_{j t}+(1-\lambda) z_{t}\right) .
\end{aligned}
$$

Denote the conditional variance by

$$
\Omega_{s}=\operatorname{var}\left(\left(\frac{1}{\theta} \varepsilon_{j t}+\frac{1-\theta}{\theta} z_{t}\right) \mid s_{j t}\right)
$$

Since $\frac{1}{\theta} \varepsilon_{j t}$ and $\frac{1-\theta}{\theta} z_{t}$ are Gaussian, the conditional variance $\Omega_{s}$ will not depend on the observed $s_{j t}$ and will be given by

$$
\Omega_{s}=\operatorname{var}\left(\frac{1}{\theta} \varepsilon_{j t}+\frac{1-\theta}{\theta} z_{t}\right)-\frac{\left(\operatorname{cov}\left(\frac{1}{\theta} \varepsilon_{j t}+\frac{1-\theta}{\theta} z_{t}, s_{j t}\right)\right)^{2}}{\operatorname{var}\left(v_{j t}+\lambda \varepsilon_{j t}+(1-\lambda) z_{t}\right)}
$$


We then have

$$
\begin{aligned}
y_{j t} & =(1-\theta) \phi_{0}+\theta \frac{\frac{1}{\theta} \lambda \sigma_{\varepsilon}^{2}+\frac{1-\theta}{\theta}(1-\lambda) \sigma_{z}^{2}}{\sigma_{v}^{2}+\lambda^{2} \sigma_{\varepsilon}^{2}+(1-\lambda)^{2} \sigma_{z}^{2}}\left(v_{j t}+\lambda \varepsilon_{j t}+(1-\lambda) z_{t}\right)+\frac{\theta}{2} \Omega_{s} \\
& \equiv \varphi_{0}+\theta \mu\left(v_{j t}+\lambda \varepsilon_{j t}+(1-\lambda) z_{t}\right)
\end{aligned}
$$

where

$$
\begin{aligned}
\mu & =\frac{\frac{1}{\theta} \lambda \sigma_{\varepsilon}^{2}+\frac{1-\theta}{\theta}(1-\lambda) \sigma_{z}^{2}}{\sigma_{v}^{2}+\lambda^{2} \sigma_{\varepsilon}^{2}+(1-\lambda)^{2} \sigma_{z}^{2}} \\
\varphi_{0} & =(1-\theta) \phi_{0}+\frac{\theta}{2} \Omega_{s}
\end{aligned}
$$

Now for equilibrium to hold we need aggregate demand to equal aggregate output. From equation (14), markets will clear if for each $z_{t}$ we have

$$
\begin{aligned}
\left(1-\frac{1}{\theta}\right)\left(\phi_{0}+z_{t}\right)= & \log \int \epsilon_{j t}^{\frac{1}{\theta}} Y_{j t}^{1-\frac{1}{\theta}} d j \\
= & \log E \exp \left[\frac{1}{\theta} \varepsilon_{t}+\left(1-\frac{1}{\theta}\right)\left[\varphi_{0}+\theta \mu\left(v_{j t}+\lambda \varepsilon_{j t}+(1-\lambda) z_{t}\right)\right]\right] \\
= & \left(1-\frac{1}{\theta}\right) \varphi_{0}+\left[\left(1-\frac{1}{\theta}\right) \theta \mu(1-\lambda)\right] z_{t} \\
& +\frac{1}{2}\left[\frac{1}{\theta}+\left(1-\frac{1}{\theta}\right) \theta \mu \lambda\right]^{2} \sigma_{\varepsilon}^{2}+\frac{1}{2}\left[\left(1-\frac{1}{\theta}\right) \theta \mu\right]^{2} \sigma_{v}^{2}
\end{aligned}
$$

Matching the coefficients yields two constraints: If $\mu \neq 0$, then

$$
\theta \mu=\frac{1}{1-\lambda}
$$

and

$$
\phi_{0}=\varphi_{0}+\frac{\theta-1}{\theta} \frac{1}{2}\left(\left(\frac{1}{\theta-1}+\theta \mu \lambda\right)^{2} \sigma_{\varepsilon}^{2}+(\theta \mu)^{2} \sigma_{v}^{2}\right)
$$

Notice $\theta \mu=\frac{1}{1-\lambda}($ when $\mu \neq 0)$ implies

$$
\theta \mu=\theta \frac{\frac{1}{\theta} \lambda \sigma_{\varepsilon}^{2}+\frac{1-\theta}{\theta}(1-\lambda) \sigma_{z}^{2}}{\sigma_{v}^{2}+\lambda^{2} \sigma_{\varepsilon}^{2}+(1-\lambda)^{2} \sigma_{z}^{2}}=\frac{1}{1-\lambda}
$$

or we have

$$
\sigma_{z}^{2}=\frac{\lambda(1-2 \lambda)}{(1-\lambda)^{2} \theta} \sigma_{\varepsilon}^{2}-\frac{1}{(1-\lambda)^{2} \theta} \sigma_{v}^{2}
$$


Notice that if either $\lambda \geq \frac{1}{2}$, or $\sigma_{v}^{2}>\lambda(1-2 \lambda) \sigma_{\varepsilon}^{2}$, then $\sigma_{z}^{2}<0$, suggesting that the only equilibrium is $z=0$. Hence, to have a self-fulfilling expectations equilibrium, we require $\lambda \in\left(0, \frac{1}{2}\right)$ and $\sigma_{v}^{2}<\lambda(1-2 \lambda) \sigma_{\varepsilon}^{2}$. This pins down $\sigma_{z}^{2}$, the variance of $z$ or of output as a function of $\sigma_{\varepsilon}^{2}$ and $\sigma_{v}^{2}$. Note that introducing the noise $v_{j t}$ into the signal makes output in the self-fulfilling equilibrium less noisy: If the signal was $s_{j t}=\lambda \varepsilon_{j t}+(1-\lambda) z_{t}$, then we would have $\sigma_{z}^{2}=\frac{\lambda(1-2 \lambda)}{(1-\lambda)^{2} \theta} \sigma_{\varepsilon}^{2}$. The reason is that the signal is now more noisy, and firms attribute a smaller fraction of the signal to demand fluctuations.

Now we consider the two constants $\phi_{0}$ and $\varphi_{0}$. First, using (A.14), we have

$$
\begin{aligned}
\Omega_{s} & =\operatorname{var}\left(\left(\frac{1}{\theta} \varepsilon_{j t}+\frac{1-\theta}{\theta} z_{t}\right) \mid s_{j t}\right) \\
& =\left[\left(\frac{1}{\theta}\right)^{2}-\frac{1}{1-\lambda} \frac{1}{\theta^{2}} \lambda\right] \sigma_{\varepsilon}^{2}+\left[\left(\frac{1-\theta}{\theta}\right)^{2}+\frac{\theta-1}{\theta^{2}}\right] \sigma_{z}^{2}
\end{aligned}
$$

Since $\sigma_{z}^{2}=\frac{\lambda(1-2 \lambda)}{(1-\lambda)^{2} \theta} \sigma_{\varepsilon}^{2}-\frac{1}{(1-\lambda)^{2} \theta} \sigma_{v}^{2}$ from (A.15), we have

$$
\Omega_{s}=\frac{(1-\lambda+(\theta-1) \lambda)(1-2 \lambda) \sigma_{\varepsilon}^{2}-(\theta-1) \sigma_{v}^{2}}{\theta^{2}(1-\lambda)^{2}}
$$

Then, from equation (A.10),

$$
\varphi_{0}=(1-\theta) \phi_{0}+\frac{1}{2 \theta} \frac{(1-\lambda+(\theta-1) \lambda)(1-2 \lambda) \sigma_{\varepsilon}^{2}-(\theta-1) \sigma_{v}^{2}}{(1-\lambda)^{2}} .
$$

From equation (A.13) we have,

$$
\phi_{0}=\varphi_{0}+\frac{\theta-1}{\theta} \frac{1}{2}\left(\left(\frac{1}{\theta-1}+\theta \mu \lambda\right)^{2} \sigma_{\varepsilon}^{2}+(\theta \mu)^{2} \sigma_{v}^{2}\right)
$$

Combining these implies

$$
\begin{aligned}
\phi_{0}= & \frac{1}{2} \frac{(1-\lambda+(\theta-1) \lambda)(1-2 \lambda) \sigma_{\varepsilon}^{2}-(\theta-1) \sigma_{v}^{2}}{\theta^{2}(1-\lambda)^{2}} \\
& +\frac{1}{2} \frac{1}{\theta-1}\left[\frac{1}{\theta}+\left(1-\frac{1}{\theta}\right) \theta \mu \lambda\right]^{2} \sigma_{\varepsilon}^{2} .
\end{aligned}
$$

Simplifying further gives,

$$
\phi_{0}=\frac{1}{2}\left(\frac{(1-\lambda+(\theta-1) \lambda)}{\theta(1-\lambda)} \frac{1}{(\theta-1)}\right) \sigma_{\varepsilon}^{2}-\frac{(\theta-1) \sigma_{v}^{2}}{2 \theta^{2}(1-\lambda)^{2}}
$$


Therefore the outputs of intermediate goods firms, conditioned on signals $s_{j t}=v_{j t}+\lambda \varepsilon_{j t}+(1-\lambda) z_{t}$, are given by

$$
y_{j t} \equiv \varphi_{0}+\theta \mu\left(v_{j t}+\lambda \varepsilon_{j t}+(1-\lambda) z_{t}\right)
$$

They constitute a market clearing stochastic rational expectations equilibrium. We now turn to the proof of Proposition 1.

2. The Fundamental Equilibrium. Firms take aggregate output as constant, so $z_{t}=0$ and $\log Y_{t}=y_{t}=\phi_{0}$, but the signal $s_{j t}=\lambda \varepsilon_{j t}+v_{j t}$ gives them imperfect information on their idiosyncratic shock. We can compute the equilibrium by setting $z_{t}=\sigma_{z}^{2}=0$, and we have

$$
\begin{gathered}
\mu=\frac{\frac{1}{\theta} \lambda \sigma_{\varepsilon}^{2}}{\sigma_{v}^{2}+\lambda^{2} \sigma_{\varepsilon}^{2}} \\
\Omega_{s}=\operatorname{var}\left(\frac{1}{\theta} \varepsilon_{j t} \mid s_{j t}\right]=\left(\frac{1}{\theta}\right)^{2}(1-\mu \theta \lambda) \sigma_{\varepsilon}^{2} \\
\varphi_{0}=(1-\theta) \phi_{0}+\frac{\theta}{2} \Omega_{s}=(1-\theta) \phi_{0}+\frac{\theta}{2}\left(\frac{1}{\theta}\right)^{2}(1-\mu \theta \lambda) \sigma_{\varepsilon}^{2} \\
\phi_{0}=\varphi_{0}+\frac{\theta-1}{\theta} \frac{1}{2}\left(\left[\frac{1}{\theta-1}+\theta \mu \lambda\right]^{2} \sigma_{\varepsilon}^{2}+(\theta \mu)^{2} \sigma_{v}^{2}\right)
\end{gathered}
$$

so that

$$
\phi_{0}=\frac{1}{2}\left[\left(\frac{\theta+\theta \mu \lambda(\theta-1)+(\theta \mu \lambda(\theta-1))^{2}}{\theta^{2}(\theta-1)}\right) \sigma_{\varepsilon}^{2}+(\theta-1)(\theta \mu)^{2} \sigma_{v}^{2}\right]
$$

\section{A. 2 Proof of Proposition 3}

Proof. In our previous case, output was equal to $y_{t}=z_{t}+\phi_{0}$. Now the agent receives two signals. The first is $s_{j t}=\lambda \varepsilon_{j t}+(1-\lambda) y_{t}^{e}+v_{j t}$, which is equivalent to $s_{j t}=\lambda \varepsilon_{j t}+(1-\lambda) z_{t}+v_{j t}$ as $\phi_{0}$ is common knowledge. The second signal is $s_{p t}=z_{t}+e_{t}$, where we can interpret $e_{t}$ as common noise in the public forecast of aggregate demand. In equilibrium we have $z_{t}=y_{t}$. Conjecture that output is equal to

$$
\log Y_{t}=y_{t}=\phi_{0}+z_{t}+\eta e_{t}
$$

where $\phi_{0}, \sigma_{z}^{2}$ and $\eta$ are constants to be determined. In that case,

$$
\operatorname{cov}\left(s_{p t}, y_{t}\right)=\sigma_{z}^{2}+\eta \sigma_{e}^{2}
$$


(Note that if $\eta=-\frac{\sigma_{z}^{2}}{\sigma_{e}^{2}}$, then this covariance term becomes zero.) The agent has two signals. The private signal is

$$
s_{j t}=\lambda \varepsilon_{j t}+(1-\lambda)\left[z_{t}+\eta e_{t}\right]+v_{j t}
$$

and the public signal is

$$
s_{p t}=z_{t}+e_{t}
$$

so we have

$$
y_{j t} \equiv(1-\theta) \phi_{0}+\theta \log E_{t}\left[\exp \left(\frac{1}{\theta} \varepsilon_{j t}+\frac{1-\theta}{\theta}\left(z_{t}+\eta e_{t}\right)\right) \mid\left\{s_{j t}, s_{p t}\right\}\right]
$$

Since the random variables are assumed normal, we can write

$$
y_{j t} \equiv(1-\theta) \phi_{0}+\frac{\theta}{2} \Omega_{s}+\theta\left[\xi_{0} s_{j t}+\xi_{1} s_{p t}\right]
$$

where $\Omega_{s}$ is the conditional variance of $x_{j t}=\frac{1}{\theta} \varepsilon_{j t}+\frac{1-\theta}{\theta}\left(z_{t}+\eta e_{t}\right)$ based on $s_{j t}$ and $s_{p t}$. Market clearing implies

$$
Y_{t}^{1-\frac{1}{\theta}}=\int_{0}^{1} \epsilon_{j t}^{\frac{1}{\theta}} Y_{j t}^{1-\frac{1}{\theta}} d j,
$$

so taking logs and equating the stochastic elements on the left and right, we must have

$$
\begin{aligned}
\frac{z_{t}+\eta e_{t}}{\theta} & =\xi_{0} \int s_{j t} d j+\xi_{1} s_{p t} \\
& =\xi_{0}(1-\lambda)\left(z_{t}+\eta e_{t}\right)+\xi_{1}\left(z_{t}+e_{t}\right)
\end{aligned}
$$

which requires

$$
\begin{aligned}
& \frac{1}{\theta}=\xi_{0}(1-\lambda)+\xi_{1} \\
& \frac{\eta}{\theta}=\xi_{0}(1-\lambda) \eta+\xi_{1}
\end{aligned}
$$

These two equations collapse to

$$
\frac{1}{\theta}=\xi_{0}(1-\lambda)
$$

We first explore the equilibrium with stochastic output where $\xi_{1}=0$. Note that the optimal solutions for $\xi_{0}$ and $\xi_{1}$ must satisfy

$$
\begin{aligned}
& E x_{j t} s_{j t}-\xi_{0} \sigma_{s_{j t}}^{2}-\xi_{1} \operatorname{cov}\left(s_{j t}, s_{p t}\right)=0 \\
& E x_{j t} s_{p t}-\xi_{0} \operatorname{cov}\left(s_{j t}, s_{p t}\right)-\xi_{1} \sigma_{s_{p t}}^{2}=0 .
\end{aligned}
$$


From (A.37) and (A.38),

$$
\xi_{0}=\frac{\lambda \frac{1}{\theta} \sigma_{\varepsilon}^{2}+(1-\lambda) \frac{1-\theta}{\theta}\left(\sigma_{z}^{2}+\eta^{2} \sigma_{e}^{2}\right)}{\lambda^{2} \sigma_{\varepsilon}^{2}+(1-\lambda)^{2}\left(\sigma_{z}^{2}+\eta^{2} \sigma_{e}^{2}\right)+\sigma_{v}^{2}}=\frac{1}{\theta} \frac{1}{1-\lambda},
$$

which yields

$$
\sigma_{z}^{2}+\eta^{2} \sigma_{e}^{2}=\frac{\lambda(1-2 \lambda)}{(1-\lambda)^{2} \theta} \sigma_{\varepsilon}^{2}-\frac{1}{\theta(1-\lambda)^{2}} \sigma_{v}^{2}
$$

Again, as stated in Proposition 3, if either $\lambda \geq \frac{1}{2}$, or $\sigma_{v}^{2}>\lambda(1-2 \lambda) \sigma_{\varepsilon}^{2}$, then $\sigma_{z}^{2}+\eta^{2} \sigma_{e}^{2} \leq 0$ and there is only the fundamental equilibrium.

Now we need to determine $\eta$. Notice that

$$
E\left(x_{j t} s_{p t}\right)=E\left[\left(\frac{1}{\theta} \varepsilon_{j t}+\frac{1-\theta}{\theta}\left(z_{t}+\eta e_{t}\right)\right) \times\left(z_{t}+e_{t}\right)\right]=\frac{1-\theta}{\theta}\left(\sigma_{z}^{2}+\eta \sigma_{e}^{2}\right)
$$

and

$$
\begin{aligned}
\operatorname{cov}\left(s_{j t}, s_{p t}\right) & =E\left(\lambda \varepsilon_{j t}+(1-\lambda)\left(z_{t}+\eta e_{t}\right)\right) \times\left(z_{t}+e_{t}\right) \\
& =(1-\lambda)\left(\sigma_{z}^{2}+\eta \sigma_{e}^{2}\right)
\end{aligned}
$$

If $\xi_{0} \neq 0$ in this case we have

$$
\sigma_{z}^{2}+\eta \sigma_{e}^{2}=0
$$

or

$$
\eta=-\frac{\sigma_{z}^{2}}{\sigma_{e}^{2}}
$$

and (A.39) is satisfied. By our assumption $\sigma_{e}^{2}=\tilde{\gamma} \sigma_{z}^{2}$ we have $\eta=-\frac{1}{\tilde{\gamma}}$. Suppose that $\lambda<\frac{1}{2}$. We have to find out whether it is possible to have a rational expectation equilibrium satisfying $\sigma_{z}^{2}>0$ . Note from (A.41) that

$$
\sigma_{z}^{2}+\eta^{2} \sigma_{e}^{2}=\frac{\lambda(1-2 \lambda)}{(1-\lambda)^{2} \theta} \sigma_{\varepsilon}^{2}-\frac{1}{\theta(1-\lambda)^{2}} \sigma_{v}^{2}
$$

Substituting $\eta$ into the expression we then have

$$
\left(\sigma_{e}^{2}\right)^{-2}\left(\sigma_{z}^{2}\right)^{2}+\sigma_{z}^{2}=\left(\frac{\lambda(1-2 \lambda)}{(1-\lambda)^{2} \theta} \sigma_{\varepsilon}^{2}-\frac{1}{\theta(1-\lambda)^{2}} \sigma_{v}^{2}\right)
$$

Using the relationship between $\sigma_{e}^{2}$ and $\sigma_{z}^{2}$ we have

$$
\frac{1+\tilde{\gamma}}{\tilde{\gamma}} \sigma_{z}^{2}=\left(\frac{\lambda(1-2 \lambda)}{(1-\lambda)^{2} \theta} \sigma_{\varepsilon}^{2}-\frac{1}{\theta(1-\lambda)^{2}} \sigma_{v}^{2}\right)
$$


Notice that the above equation has an unique solution for $\sigma_{z}^{2}>0$ :

$$
\sigma_{z}^{2}=\frac{1+\tilde{\gamma}}{\tilde{\gamma}}\left(\frac{\lambda(1-2 \lambda)}{(1-\lambda)^{2} \theta} \sigma_{\varepsilon}^{2}-\frac{1}{\theta(1-\lambda)^{2}} \sigma_{v}^{2}\right)
$$

If $\tilde{\gamma}$ approaches zero, $\sigma_{z}^{2}$ also approaches to zero. However, since $\sigma_{e}^{2}=\tilde{\gamma} \sigma_{z}^{2}$ and $\eta=-\frac{1}{\tilde{\gamma}}$, the variance of output is given by

$$
\sigma_{y}^{2}=\frac{1+\tilde{\gamma}}{\tilde{\gamma}} \sigma_{z}^{2}=\left(\frac{\lambda(1-2 \lambda)}{(1-\lambda)^{2} \theta} \sigma_{\varepsilon}^{2}-\frac{1}{\theta(1-\lambda)^{2}} \sigma_{v}^{2}\right)
$$

which is not affected and the uncertainty equilibrium will continue to exist.

Finally, since the public signal is not informative at all, the firm's effective signal is only the private one. We can redefine

$$
\hat{z}_{t}=z_{t}+\eta e_{t}=z_{t}-\frac{1}{\tilde{\gamma}} e_{t}
$$

which then has variance

$$
\sigma_{\hat{z}}^{2}=\frac{\lambda(1-2 \lambda)}{(1-\lambda)^{2} \theta} \sigma_{\varepsilon}^{2}-\frac{1}{\theta(1-\lambda)^{2}} \sigma_{v}^{2}
$$

where we again use $\sigma_{e}^{2}=\tilde{\gamma} \sigma_{z}^{2}$ and $\eta=-\frac{1}{\tilde{\gamma}}$ to derive (A.52). So output will be as in Proposition 3,

$$
y_{t}=z_{t}+\eta e_{t}+\phi_{0}=\hat{z}_{t}+\phi_{0}
$$

where the constant term is $\phi_{0}=\frac{1}{2}\left(\frac{(1-\lambda+(\theta-1) \lambda)}{\theta(1-\lambda)} \frac{1}{(\theta-1)}\right) \sigma_{\varepsilon}^{2}-\frac{(\theta-1) \sigma_{v}^{2}}{2 \theta^{2}(1-\lambda)^{2}}$. With $z_{t}$ redefined as $\hat{z}_{t}$, the property of output fluctuations is not affected.

We now turn to the fundamental equilibrium. From (A.35) and (A.36), if $\xi_{1} \neq 0$, we must have $\eta=1$. Namely aggregate output will be

$$
y_{t}=\phi_{0}+z_{t}+e_{t}
$$

If the public signal is still as $s_{p t}=z_{t}+e_{t}$ it fully reveals aggregate demand $y_{t}$. The private signal would now be $s_{j t}=\lambda \varepsilon_{j t}+(1-\lambda)\left[z_{t}+e_{t}\right]+v_{j t}=\lambda \varepsilon_{j t}+(1-\lambda)\left[\left(y_{t}-\phi_{0}\right)\right]+v_{j t}$ where by construction $y_{t}-\phi_{0}$ will be known. If we define $\hat{z}_{t}=z_{t}+e_{t}$, and attempt to define an equilibrium analogous to the equilibrium of Proposition 1, with the difference that the aggregate demand shock $\hat{z}_{t}=z_{t}+e_{t}$ is not taken as zero but is perfectly observed each period prior to the production decision, we reach a contradiction. Setting $z_{t}=0$, the "constant" term $\phi_{0}$ can be defined to include $e_{t}$ and solved as in Proposition 1 as a function of time-invariant parameters of the model. However this will contradict the randomness of $e_{t}$ unless $e_{t}=0$ for all $t$. The fundamental equilibrium of Proposition 1 with 
constant output is not compatible with a time-varying public forecast of aggregate demand since firms would forecast the constant output. The public signal $s_{p t}=z_{t}+e_{t}$ would be observed in the self-fulfilling equilibrium, but in the fundamental equilibrium the public forecast of aggregate output would be a constant, and identical to the equilibrium in Proposition 1. If on the other hand we use our assumption that the variance of the forecast error of the public signal is proportional to the variance of $z$, that is if $\sigma_{e}^{2}=\tilde{\gamma} \sigma_{z}^{2}$, then we can recover the fundamental equilibrium of Proposition 1 where output is constant: for this equilibrium we would have $z_{t}=e_{t}=0$ for all $t$. 